\title{
EXISTENCE OF A SOLUTION OF THE MIXED FORMULATION FOR GENERALIZED FORCHHEIMER FLOWS OF ISENTROPIC GASES
}

\author{
THINH KIEU ${ }^{\dagger}$
}

\begin{abstract}
This paper is focused on the generalized Forchheimer flows of isentropic gas, described by a system of two nonlinear degenerating differential equations of first order. We prove the existence and uniqueness of the Dirichlet problem for stationary problem. The technique of semi-discretization in time is used to prove the existence for the time-dependent problem.
\end{abstract}

Key words. Porous media, isentropic gas, slightly compressible fluids, generalized Forchheimer equations, existence.

AMS subject classifications. 35Q35, 35D30, 35K55, 76S05.

1. Introduction. We consider a fluid in porous medium occupying a bounded domain $\Omega \subset \mathbb{R}^{d}, d \geq 2$ with boundary $\Gamma$. Let $x \in \mathbb{R}^{d}, 0<T<\infty$ and $t \in(0, T]$ be the spatial and time variables respectively. The fluid flow has velocity $v(x, t) \in \mathbb{R}^{d}$, pressure $p(x, t) \in \mathbb{R}$ and density $u(x, t) \in \mathbb{R}_{+}$.

The Darcy-Forchheimer equation, which is considered as a momentum equation, is studied in [2,8-10] of the form

$$
-\nabla p=\sum_{i=0}^{N} a_{i}|v|^{\alpha_{i}} v .
$$

In order to take into account the presence of density in generalized Forchheimer equation, we modify (1.1) using dimension analysis by Muskat [20] and Ward [24]. They proposed the following equation for both laminar and turbulent flows in porous media:

$$
-\nabla p=G\left(v^{\alpha} \kappa^{\frac{\alpha-3}{2}} \rho^{\alpha-1} \mu^{2-\alpha}\right), \text { where } G \text { is a function of one variable. }
$$

In particular, when $\alpha=1,2$, Ward [24] established from experimental data that

$$
-\nabla p=\frac{\mu}{\kappa} v+c_{F} \frac{\rho}{\sqrt{\kappa}}|v| v, \quad \text { where } c_{F}>0 .
$$

Combining (1.1) with the suggestive form (1.2) for the dependence on $\rho$ and $v$, we propose the following equation

$$
-\nabla p=\sum_{i=0}^{N} a_{i} \rho^{\alpha_{i}}|v|^{\alpha_{i}} v,
$$

where $N \geq 1, \alpha_{0}=0<\alpha_{1}<\ldots<\alpha_{N}$ are fixed real numbers, the coefficients $a_{0}(x, t), \ldots, a_{N}(x, t)$ are non-negative with $0<\underline{\mathrm{a}}<a_{0}(x, t), a_{N}(x, t)<\bar{a}<\infty, 0 \leq a_{i}(x, t) \leq \bar{a}<\infty, i=1, \ldots, N-1$.

Multiplying both sides of the equation (1.4) to $\rho$, we find that

$$
\left(\sum_{i=0}^{N} a_{i}|\rho v|^{\alpha_{i}}\right) \rho v=-\rho \nabla p .
$$

\footnotetext{
${ }_{\dagger}^{\dagger}$ Department of Mathematics, University of North Georgia, Gainesville Campus, 3820 Mundy Mill Rd., Oakwood, GA 30566, U.S.A. (thinh.kieu@ung.edu).
} 
Denote the function $F: \Omega \times[0, T] \times \mathbb{R}^{+} \rightarrow \mathbb{R}^{+}$a generalized polynomial with non-negative coefficients by

$$
F(x, t, z)=a_{0}(x, t) z^{\alpha_{0}}+a_{1}(x, t) z^{\alpha_{1}}+\cdots+a_{N}(x, t) z^{\alpha_{N}}, \quad z \geq 0
$$

The equation (1.5) can be rewritten as

$$
F(x, t,|\rho v|) \rho v=-\rho \nabla p .
$$

For isentropic gases, the constitutive law is

$$
p=c \rho^{\gamma} \quad \text { for some } c, \gamma>1 \text {. }
$$

Then from (1.7) and (1.8) follows

$$
F(x, t,|\rho v|) \rho v=-\rho \nabla p=-\nabla u \quad \text { with } u=\frac{c \gamma \rho^{\gamma+1}}{\gamma+1} .
$$

The continuity equation is

$$
\phi(x) \partial_{t} \rho+\operatorname{div}(\rho v)=f(x, t)
$$

where $\phi$ is the porosity, $f$ is external mass flow rate .

Rewrite

$$
\rho=\left(\frac{\gamma+1}{c \gamma}\right)^{\frac{1}{\gamma+1}} u^{\lambda} \quad \text { with } \lambda=\frac{1}{\gamma+1} \in(0,1) .
$$

Combining (1.10) with relation (1.11), we have

$$
\phi(x)\left(\frac{\gamma+1}{c \gamma}\right)^{\lambda} \partial_{t} u^{\lambda}+\operatorname{div}(\rho v)=f(x, t) .
$$

By combining (1.9) and (1.10) we have

$$
\begin{array}{r}
F(x, t,|\mathbf{m}|) \mathbf{m}=-\nabla u, \\
\phi(x)\left(\frac{\gamma+1}{c \gamma}\right)^{\lambda} \partial_{t} u^{\lambda}+\operatorname{div} \mathbf{m}=f(x, t),
\end{array}
$$

where $\mathbf{m}=\rho v$.

By rescaling the variable $\phi(x) \rightarrow\left(\frac{\gamma+1}{c \gamma}\right)^{\lambda} \phi(x)$. We obtain system of equations

$$
\begin{aligned}
F(x, t,|\mathbf{m}|) \mathbf{m} & =-\nabla u, \\
\phi(x) \partial_{t} u^{\lambda}+\operatorname{div} \mathbf{m} & =f(x, t) .
\end{aligned}
$$

The Darcy- Forchheimer equation in (1.13) leads to

$$
\mathcal{F}(|\mathbf{m}|)=F(x, t,|\mathbf{m}|)|\mathbf{m}|=|\nabla u|, \quad \text { where } \mathcal{F}(s)=s F(s) .
$$

Since $\mathcal{F}$ is a one-to-one mapping from $[0, \infty)$ onto $[0, \infty)$, therefore one can find a unique non-negative $|\mathbf{m}|$ as a function of $|\nabla u|$,

$$
|\mathbf{m}|=\mathcal{F}^{-1}(|\nabla u|)
$$


Solving for $\mathbf{m}$ from the first equation of (1.13) gives

$$
\mathbf{m}=-\frac{\nabla u}{F\left(x, t, \mathcal{F}^{-1}(|\nabla u|)\right)}=-K(x, t,|\nabla u|) \nabla u,
$$

where the function $K: \Omega \times[0, T] \times \mathbb{R}^{+} \rightarrow \mathbb{R}^{+}$is defined for $\xi \geq 0$ by

$$
K(x, t, \xi)=\frac{1}{F(x, t, s(x, t, \xi))},
$$

with $s=s(x, t, \xi)$ being the unique non-negative solution of $s F(s)=\xi$.

Note that

$$
\mathcal{F}^{-1}(0)=0, \quad K(x, t, 0)=\frac{1}{F(x, t, 0)}=\frac{1}{a_{0}(x, t)}>0
$$

Substituting (1.14) into the second equation of (1.13) we obtain a scalar partial differential equation (PDE) for the density:

$$
\phi(x) \partial_{t} u^{\lambda}-\operatorname{div}(K(x, t,|\nabla u|) \nabla u)=f(x, t), \quad(x, t) \in \Omega \times[0, T] .
$$

From mathematical point of view, equation 1.16 for $\lambda<1$ is a doubly nonlinear parabolic equation, which is an interesting topic of its own. Research on doubly nonlinear parabolic equations follows the development of general parabolic equations [16, 18] and degenerate/singular parabolic equations [6,7] (see also the treaties in [12, 16-18].) However, it requires much more complicated techniques. See monograph [12], review paper [11,13] and references therein.

In the this paper, we focus on proving the existence of weak solutions of the system (1.13) for the Dirichlet boundary conditions with general coefficient functions, while imposing only minimal regularity assumptions. Such a problem was not studied in the literature previously. Our proof of solvability is based on the stationary problem first by applying the technique of the theory of nonlinear monotone operators (e.g., in [3, 19, 23, 25]) to prove the existence and uniqueness of a weak solution of the corresponding elliptic problem of (1.13). Then, using the technique of semi-discretization in time (see e.g., [1, 14, 21]), we prove the existence of weak solutions of the parabolic problem by constructing approximate solutions. This approach can be extend in straightforward way to time-dependent nonlinear problems with degenerate coefficients or doubly nonlinear parabolic equations.

The paper is organized as follows. Section $\$ 2$ contains a brief summary of some notations and the relevant results. In Section $\$ 3$, we consider the stationary problem of (1.13). The existence and uniqueness of a solution are proved in Theorem 3.1. Section \$4 is intended to motivate our investigation of the semi-discrete problem after discretization of the time-derivative in (1.13) and show again the existence and uniqueness of a solution in Theorem 4.3. Section $\$ 5$ is devoted to the study of the transient problem governed by (5.1) with homogeneous boundary conditions. We derive a priori estimates of the solutions to (5.2). These are used to prove the solvability of the transient problem (5.1).

2. Notations and preliminary results. Through out this paper, we assume that $\Omega$ is an open, bounded subset of $\mathbb{R}^{d}$, with $d=2,3, \ldots$, and has $C^{1}$-boundary $\partial \Omega$. For $s \in[0, \infty)$, we denote $L^{s}(\Omega)$ be the set of s-integrable functions on $\Omega$ and $\left(L^{s}(\Omega)\right)^{d}$ the space of $d$-dimensional vectors which have all components in $L^{s}(\Omega)$. We denote $(\cdot, \cdot)$ the inner product in either $L^{s}(\Omega)$ or $\left(L^{s}(\Omega)\right)^{d}$ that is $(\xi, \eta)=\int_{\Omega} \xi \eta d x$ 
or $(\boldsymbol{\xi}, \boldsymbol{\eta})=\int_{\Omega} \xi \cdot \boldsymbol{\eta} d x$ and $\|u\|_{L^{s}(\Omega)}=\left(\int_{\Omega}|u(x)|^{s} d x\right)^{1 / s}$ for standard Lebesgue norm of the measurable function. The notation $\langle\cdot, \cdot\rangle$ will be used for the $L^{s}(\partial \Omega)$ inner-product. For $m \geq 0, s \in[0, \infty]$, we denote the Sobolev spaces by $W^{m, s}(\Omega)=\left\{v \in L^{s}(\Omega): D^{\alpha} v \in L^{s}(\Omega),|\alpha| \leq m\right\}$ and the norm of $W^{m, s}(\Omega)$

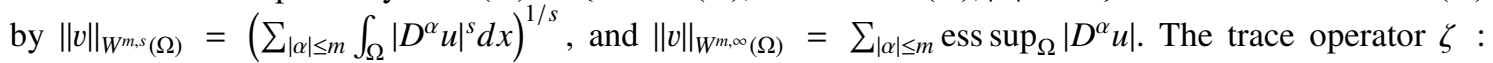
$W^{m, s}(\Omega) \rightarrow W^{m-1 / s, s}(\partial \Omega)$ is onto. We denote by $W_{0}^{m, s}(\Omega)$ the kernel of $\zeta$ and its dual space by $W^{-m, s^{*}}(\Omega)=$ $\left(W_{0}^{m, s}(\Omega)\right)^{\prime}$, where $1 / s+1 / s^{*}=1$. The test space $\mathcal{D}(\Omega):=C_{0}^{\infty}(\Omega)$ is dense subset of $L^{s}(\Omega)$ and of $W_{0}^{m, s}(\Omega)$ and that of $\mathcal{D}(\bar{\Omega}):=\left\{\varphi, \varphi \in \mathcal{D}\left(\mathbb{R}^{n}\right)\right\}$ is a dense subspace of $W^{m, s}(\Omega)$. Finally we define $L^{s}(0, T ; X)$ to be the space of all measurable functions $v:[0, T] \rightarrow X$ with the norm $\|v\|_{L^{s}(0, T ; X)}=\left(\int_{0}^{T}\|v(t)\|_{X}^{s} d t\right)^{1 / s}$, and $L^{\infty}(0, T ; X)$ to be the space of all measurable functions $v:[0, T] \rightarrow X$ such that $v: t \rightarrow\|v(t)\|_{X}$ is essentially bounded on $[0, T]$ with the norm $\|v\|_{L^{\infty}(0, T ; X)}=\operatorname{ess}_{\sup _{t \in[0, T]}}\|v(t)\|_{X}$.

Our calculations frequently use the following exponents

$$
\begin{aligned}
& s=\alpha_{N}+2, \quad \alpha=\frac{\alpha_{N}}{\alpha_{N}+1}, \quad s^{*}=2-\alpha=\frac{s}{s-1}, \\
& r=1+\lambda \in(1,2), \quad r^{*}=\frac{\lambda+1}{\lambda}=1+\frac{1}{\lambda} \in(2, \infty) .
\end{aligned}
$$

The arguments $C, C_{1}, C_{2} \ldots$ represent for positive generic constants and their values depend on exponents, coefficients of polynomial $F$, the spatial dimension $d$ and domain $\Omega$, independent of the initial and boundary data and time step. These constants may be different place by place.

We introduce the space $W(\operatorname{div} ; \Omega)$ defined by $W(\operatorname{div} ; \Omega)=\left\{\mathbf{v} \in\left(\mathrm{L}^{\mathrm{s}}(\Omega)\right)^{\mathrm{d}}, \nabla \cdot \mathbf{v} \in \mathrm{L}^{\mathrm{r}^{*}}(\Omega)\right\}$ and equipped it with the norm $\|\mathbf{v}\|_{W(\operatorname{div} ; \Omega)}=\|\mathbf{v}\|_{L^{s}}+\|\nabla \cdot \mathbf{v}\|_{L^{*}}$. Since $W(\operatorname{div} ; \Omega)$ is a closed subspace of $\left(L^{s}(\Omega)\right)^{d}$, it follows that $W(\operatorname{div} ; \Omega)$ is a reflexive Banach space; the boundary $\left.\mathbf{v} \cdot v\right|_{\partial \Omega}$ exist and belong to $W^{-1 / s, s^{*}}(\partial \Omega)$ and we have the Green's formula

$$
\int_{\Omega} \mathbf{v} \nabla \psi d x+\int_{\Omega} \psi \nabla \cdot \mathbf{v} d x=\int_{\partial \Omega} \psi \mathbf{v} \cdot v d \sigma
$$

hold for every $\mathbf{v} \in W(\operatorname{div} ; \Omega)$ and $\psi \in(W(\operatorname{div} ; \Omega))^{\prime}$ (see Lemma 3 in [4]).

The function $F(\cdot)$ has the following properties.

Lemma 2.1. The following inequality hold for all $y^{\prime}, y \in \mathbb{R}^{d}$

$$
\begin{aligned}
\text { (i) } & \left|F\left(x, t,\left|y^{\prime}\right|\right) y^{\prime}-F(x, t,|y|) y\right| \leq C_{1}\left(1+\left|y^{\prime}\right|^{\alpha_{N}}+|y|^{\alpha_{N}}\right)\left|y^{\prime}-y\right| . \\
\text { (ii) } & \left(F\left(x, t,\left|y^{\prime}\right|\right) y^{\prime}-F(x, t,|y|) y\right) \cdot\left(y^{\prime}-y\right) \geq C_{2}\left(\left|y^{\prime}-y\right|^{2}+\left|y^{\prime}-y\right|^{s}\right),
\end{aligned}
$$

where the constants $C_{1}(N, \bar{a}, \operatorname{deg}(\mathrm{F}))>0$, and $C_{2}(N, \underline{a}, \operatorname{deg}(\mathrm{F}))>0$.

Proof.

(i) Let $\gamma(t)=\tau y^{\prime}+(1-\tau) y, \tau \in[0,1]$ and $h(t)=F(x, t,|\gamma(\tau)|) \gamma(\tau)$. Then

$$
\begin{aligned}
\mid F(x, & \left.t,\left|y^{\prime}\right|\right) y^{\prime}-F(x, t,|y|) y|=| h(1)-h(0)|=| \int_{0}^{1} h^{\prime}(\tau) d \tau \mid \\
& =\left|\int_{0}^{1} F(x, t,|\gamma(\tau)|)\left(y^{\prime}-y\right)+F_{z}(x, t,|\gamma(\tau)|) \frac{\gamma(\tau)\left(y^{\prime}-y\right)}{|\gamma(\tau)|} \gamma(\tau) d \tau\right| \\
& \leq\left|y^{\prime}-y\right| \int_{0}^{1} F(x, t,|\gamma(t)|)+F_{z}(x, t,|\gamma(\tau)|)|\gamma(\tau)| d \tau .
\end{aligned}
$$


Note that $F_{z}(x, t,|\gamma(\tau)|)|\gamma(\tau)|=\sum_{i=0}^{N} a_{i} \alpha_{i}|\gamma(\tau)|^{\alpha_{i}} \leq \alpha_{N} F(x, t,|\gamma(\tau)|)$ thus

$$
\left|F\left(x, t,\left|y^{\prime}\right|\right) y^{\prime}-F(x, t,|y|) y\right| \leq\left(1+\alpha_{N}\right)\left|y^{\prime}-y\right| \int_{0}^{1} F(x, t,|\gamma(\tau)|) d \tau .
$$

Using the inequality $x^{\beta} \leq 1+x^{\gamma}$ for $x \geq 0,0<\beta<\gamma$ we find that

$$
F(x, t, s) \leq \max _{i=0, \ldots, N} a_{i}(x, t) \sum_{i=0}^{N} 1+s^{\alpha_{N}} \leq(N+1) \max _{i=0, \ldots, N} a_{i}(x, t)\left(1+s^{\alpha_{N}}\right) .
$$

Thus

$$
\begin{aligned}
\left|F\left(x, t,\left|y^{\prime}\right|\right) y^{\prime}-F(x, t,|y|) y\right| & \leq\left(1+\alpha_{N}\right)(N+1) \max _{i=0, \ldots, N} a_{i}\left|y^{\prime}-y\right|\left(1+\int_{0}^{1}|\gamma(\tau)|^{\alpha_{N}} d \tau\right) \\
& \leq\left(1+\alpha_{N}\right)(N+1) \max _{i=0, \ldots, N} a_{i}\left|y^{\prime}-y\right|\left(1+\int_{0}^{1}\left(\left|y^{\prime}\right|+|y|\right)^{\alpha_{N}} d \tau\right) \\
& \leq 2^{\alpha_{N}}\left(1+\alpha_{N}\right)(N+1) \max _{i=0, \ldots, N} a_{i}\left(1+\left|y^{\prime}\right|^{\alpha_{N}}+|y|^{\alpha_{N}}\right)\left|y^{\prime}-y\right|,
\end{aligned}
$$

which proves (2.4) hold.

(ii) Let $k(\tau)=F(x, t,|\gamma(\tau)|) \gamma(\tau)\left(y^{\prime}-y\right)$. Then

$$
\begin{aligned}
& \left(F\left(x, t,\left|y^{\prime}\right|\right) y^{\prime}-F(x, t,|y|) y\right) \cdot\left(y^{\prime}-y\right)=k(1)-k(0)=\int_{0}^{1} k^{\prime}(\tau) d \tau \\
& \quad=\int_{0}^{1}\left(F(x, t,|\gamma(\tau)|)\left|y^{\prime}-y\right|^{2}+F_{z}(x, t,|\gamma(\tau)|) \frac{\left|\gamma(\tau)\left(y^{\prime}-y\right)\right|^{2}}{|\gamma(\tau)|}\right) d \tau .
\end{aligned}
$$

Note that

$$
\frac{\left|\gamma(\tau)\left(y^{\prime}-y\right)\right|^{2}}{|\gamma(\tau)|}=\frac{|\gamma(\tau)|^{2}\left|y^{\prime}-y\right|^{2} \cos ^{2}(\beta(t))}{|\gamma(\tau)|}=|\gamma(t)|\left|y^{\prime}-y\right|^{2} \cos ^{2}(\beta(t))
$$

where $\beta(t)$ is angle between $\gamma(t)$ and $y^{\prime}-y$. It implies that

$$
\begin{aligned}
(F(x, & \left.\left.t,\left|y^{\prime}\right|\right) y^{\prime}-F(x, t,|y|) y\right) \cdot\left(y^{\prime}-y\right) \\
& =\left|y^{\prime}-y\right|^{2} \int_{0}^{1}\left(F(x, t,|\gamma(\tau)|)+F_{z}(x, t,|\gamma(\tau)|)|\gamma(\tau)| \cos ^{2}(\beta(t)) d \tau\right. \\
& \geq\left|y^{\prime}-y\right|^{2} \int_{0}^{1}\left(1+\alpha_{1} \cos ^{2}(\beta(t)) F(x, t,|\gamma(\tau)|) d \tau\right. \\
& \geq\left|y^{\prime}-y\right|^{2}\left(a_{0}+a_{N} \int_{0}^{1}|\gamma(t)|^{\alpha_{N}} d t\right) .
\end{aligned}
$$

The two last inequality are obtained by using the inequalities

$$
F_{z}(x, t,|\gamma(\tau)|)|\gamma(\tau)| \geq \alpha_{1} F(x, t,|\gamma(\tau)|) \quad \text { and } \quad F(x, t,|\gamma(\tau)|) \geq a_{0}+a_{N}|\gamma(\tau)|^{\alpha_{N}}
$$

It is proved (see e.g in [5] Lemma 2.4) that

$$
\int_{0}^{1}|\gamma(t)|^{\alpha_{N}} d t \geq \frac{\left|y^{\prime}-y\right|^{\alpha_{N}}}{2^{\alpha_{N}+1}\left(\alpha_{N}+1\right)}
$$


Hence

$$
\left(F\left(x, t,\left|y^{\prime}\right|\right) y^{\prime}-F(x, t,|y|) y\right) \cdot\left(y^{\prime}-y\right) \geq\left(1+\alpha_{1}\right)\left|y^{\prime}-y\right|^{2}\left(a_{0}+a_{N} \frac{\left|y^{\prime}-y\right|^{\alpha_{N}}}{2^{\alpha_{N}+1}\left(\alpha_{N}+1\right)}\right)
$$

The proof is complete.

We recall some elementary inequalities that will be used in this paper.

Lemma 2.2. The following inequality hold for all $a, b \geq 0, \lambda \in(0,1]$.

$$
\begin{aligned}
& \frac{a^{p}+b^{p}}{2} \leq(a+b)^{p} \leq 2^{|p-1|}\left(a^{p}+b^{p}\right) \quad \text { for all } p>0 \\
& \left|a^{\lambda}-b^{\lambda}\right| \leq|a-b|^{\lambda} \\
& \frac{|a-b|^{2}}{|a|^{1-\lambda}+|b|^{1-\lambda}} \leq\left(a^{\lambda}-b^{\lambda}\right)(a-b) \\
& \left(a^{\lambda}-b^{\lambda}\right) a \geq \frac{\lambda}{1+\lambda}\left(a^{1+\lambda}-b^{1+\lambda}\right)=\frac{1}{r^{*}}\left(a^{r}-b^{r}\right)
\end{aligned}
$$

3. The steady-state problem. We consider the stationary problem governed by the Darcy-Forchheimer equation and the stationary continuity equation together with Dirichlet boundary condition

$$
\begin{array}{rr}
F(x,|\mathbf{m}|) \mathbf{m}=-\nabla u & x \in \Omega, \\
\operatorname{div} \mathbf{m}=f(x) & x \in \Omega, \\
u=-u_{b}(x) & x \in \partial \Omega .
\end{array}
$$

3.1. The mixed formulation of the stationary problem. The mixed formulation of (3.1) reads as follows. Find $(\mathbf{m}, u) \in W(\operatorname{div} ; \Omega) \times \mathrm{L}^{\mathrm{r}}(\Omega)$ such that

$$
\begin{array}{r}
(F(x,|\mathbf{m}|) \mathbf{m}, \mathbf{v})-(u, \nabla \cdot \mathbf{v})=-\left\langle u_{b}, \mathbf{v} \cdot v\right\rangle \quad \text { for all } \mathbf{v} \in W(\operatorname{div} ; \Omega), \\
(\nabla \cdot \mathbf{m}, q)=(f, q) \quad \text { for all } q \in L^{r}(\Omega) .
\end{array}
$$

We introduce a bilinear form $b: W(\operatorname{div} ; \Omega) \times \mathrm{L}^{\mathrm{r}}(\Omega) \rightarrow \mathbb{R}$ by mean of

$$
b(\mathbf{v}, q)=(\nabla \cdot \mathbf{v}, q) \quad \text { for all } \mathbf{v} \in W(\operatorname{div} ; \Omega), \mathrm{q} \in \mathrm{L}^{\mathrm{r}}(\Omega)
$$

and a nonlinear form $a:\left(L^{s}(\Omega)\right)^{d} \times\left(L^{s}(\Omega)\right)^{d} \rightarrow \mathbb{R}$ by mean of

$$
a(\mathbf{u}, \mathbf{v})=(F(x, t,|\mathbf{u}|) \mathbf{u}, \mathbf{v}) \quad \text { for all } \mathbf{u}, \mathbf{v} \in\left(L^{s}(\Omega)\right)^{d}
$$

Then we rewrite the mixed formulation $(3.2)$ as follows. Find $(\mathbf{m}, u) \in W(\operatorname{div}, \Omega) \times \mathrm{L}^{\mathrm{r}}(\Omega) \equiv \mathrm{V} \times \mathrm{Q}$ such that

$$
\begin{array}{r}
a(\mathbf{m}, \mathbf{v})-b(\mathbf{v}, u)=-\left\langle u_{b}, \mathbf{v} \cdot v\right\rangle \quad \text { for all } \mathbf{v} \in W(\operatorname{div} ; \Omega) \\
b(\mathbf{m}, q)=(f, q) \quad \text { for all } q \in Q
\end{array}
$$

3.2. Existence results. This subsection is devoted to establish the existence and uniqueness of weak solution of the stationary problem 3.1 .

Theorem 3.1. Suppose $f \in L^{r^{*}}(\Omega)$, and $u_{b} \in W^{1 / s, s}(\partial \Omega)$. The mixed formulation (3.2) of the stationary problem (3.1) has a unique solution $(\mathbf{m}, u) \in W(\operatorname{div} ; \Omega) \times \mathrm{L}^{\mathrm{r}}(\Omega)$. 
Proof. We use regularization to show the existence of a weak solution $(\mathbf{m}, u) \in V \times Q$ to problem (3.2). The proof will be divided into four steps. In step 1, we introduce an approximate problem. In step 2 we show that the approximate solution $\left(\mathbf{m}_{\varepsilon}, u_{\varepsilon}\right)$ is bounded independence of $\varepsilon$. In step 3 we prove the limit $(\mathbf{m}, u)$ of the approximate solution $\left(\mathbf{m}_{\varepsilon}, u_{\varepsilon}\right)$ satisfying problem (3.2). Step 4 is devoted to prove the uniqueness of weak solution $(\mathbf{m}, u)$ to the problem (3.2).

Step 1. For the fixed $\varepsilon>0$, we consider the following regularized problem. Find $\left(\mathbf{m}_{\varepsilon}, u_{\varepsilon}\right) \in V \times Q$ such that

$$
\begin{aligned}
& a\left(\mathbf{m}_{\varepsilon}, \mathbf{v}\right)+\varepsilon\left(\left|\nabla \cdot \mathbf{m}_{\varepsilon}\right|^{r^{*}-2} \nabla \cdot \mathbf{m}_{\varepsilon}, \nabla \cdot \mathbf{v}\right)-b\left(\mathbf{v}, u_{\varepsilon}\right)=-\left\langle u_{b}, \quad \mathbf{v} \cdot v\right\rangle \quad \text { for all } \mathbf{v} \in V, \\
& \varepsilon\left(u_{\varepsilon}^{\lambda}, q\right)+b\left(\mathbf{m}_{\varepsilon}, q\right)=(f, q) \quad \text { for all } q \in Q .
\end{aligned}
$$

Lemma 3.2. For every $\varepsilon>0$, there is a unique solution $\left(\mathbf{m}_{\varepsilon}, u_{\varepsilon}\right) \in V \times Q$ of the regularized problem (3.4).

Proof. Adding the left hand side of (3.4), we obtain the nonlinear form defined on $V \times Q$,

$a_{\varepsilon}\left(\left(\mathbf{m}_{\varepsilon}, u_{\varepsilon}\right),(\mathbf{v}, q)\right) \stackrel{\text { def }}{=} a\left(\mathbf{m}_{\varepsilon}, \mathbf{v}\right)+\varepsilon\left(\mid \nabla \cdot \mathbf{m}_{\varepsilon} r^{r^{*}-2} \nabla \cdot \mathbf{m}_{\varepsilon}, \nabla \cdot \mathbf{v}\right)-b\left(\mathbf{v}, u_{\varepsilon}\right)+\varepsilon\left(u_{\varepsilon}^{\lambda}, q\right)+b\left(\mathbf{m}_{\varepsilon}, q\right)$ for all $(\mathbf{v}, q) \in V \times Q$.

A nonlinear operator $\mathcal{A}_{\varepsilon}: V \times Q \rightarrow(V \times Q)^{\prime}$ defined by

$$
\left\langle\mathcal{A}_{\varepsilon}((\mathbf{u}, p)),(\mathbf{v}, q)\right\rangle_{(V \times Q)^{\prime} \times(V \times Q)}=a_{\varepsilon}((\mathbf{u}, p),(\mathbf{v}, q)) .
$$

Then $\mathcal{A}_{\varepsilon}$ is continuous, coercive and strictly monotone.

Applying the theorem of Browder and Minty (see in [26], Thm. 26.A) for every $\tilde{f} \in(V \times Q)^{\prime}$, there exists unique a solution $\left(\mathbf{m}_{\varepsilon}, u_{\varepsilon}\right) \in V \times Q$ of the operator equation $\mathcal{A}_{\varepsilon}\left(\mathbf{m}_{\varepsilon}, u_{\varepsilon}\right)=\tilde{f}$. In particular, we choose the linear form $\tilde{f}$ defined by $\tilde{f}(\mathbf{v}, q):=-\left\langle u_{b}, \mathbf{v} \cdot v\right\rangle+(f, q)$, which arises by adding the right hand sides of (3.4). Therefore (3.4) has a unique solution.

What is left is to show that $\mathcal{A}_{\varepsilon}$ is continuous, coercive and strictly monotone.

For the continuity,

$$
\begin{aligned}
& \left\langle\mathcal{A}_{\varepsilon}\left(\left(\mathbf{u}_{1}, p_{1}\right)-\mathcal{A}_{\varepsilon}\left(\left(\mathbf{u}_{2}, p_{2}\right)\right),(\mathbf{v}, q)\right\rangle_{(V \times Q)^{\prime} \times(V \times Q)}=a\left(\mathbf{u}_{1}, \mathbf{v}\right)-a\left(\mathbf{u}_{2}, \mathbf{v}\right)\right. \\
& \quad+\varepsilon\left(\left|\nabla \cdot \mathbf{u}_{1}\right|^{r^{*}-2} \nabla \cdot \mathbf{u}_{1}-\left|\nabla \cdot \mathbf{u}_{2}\right|^{r^{*}-2} \nabla \cdot \mathbf{u}_{2}, \nabla \cdot \mathbf{v}\right)+\varepsilon\left(p_{1}^{\lambda}-p_{2}^{\lambda}, q\right)-b\left(\mathbf{v}, p_{1}-p_{2}\right)+b\left(\mathbf{u}_{1}-\mathbf{u}_{2}, q\right) .
\end{aligned}
$$

By (2.4) and using Hölder's inequality

$$
\begin{aligned}
a\left(\mathbf{u}_{1}, \mathbf{v}\right)-a\left(\mathbf{u}_{2}, \mathbf{v}\right) & \leq\left(\left(1+\left|\mathbf{u}_{1}\right|^{s-2}+\left|\mathbf{u}_{2}\right|^{s-2}\right)\left|\mathbf{u}_{1}-\mathbf{u}_{2}\right|,|\mathbf{v}|\right) \\
& \leq C\left(1+\left\|\mathbf{u}_{1}\right\|_{L^{s}}^{s-2}+\left\|\mathbf{u}_{2}\right\|_{L^{s}}^{s-2}\right)\left\|\mathbf{u}_{1}-\mathbf{u}_{2}\right\|_{L^{s}} .
\end{aligned}
$$

On account of (2.7) and using Hölder's inequality

$$
\begin{aligned}
& \left(p_{1}^{\lambda}-p_{2}^{\lambda}, q\right) \leq\left(\left|p_{1}-p_{2}\right|^{\lambda}, q\right) \leq\left\|p_{1}-p_{2}\right\|_{L^{r}}^{\lambda}\|q\|_{L^{r}}, \\
& b\left(\mathbf{v}, p_{1}-p_{2}\right) \leq\|\nabla \cdot \mathbf{v}\|_{L^{*}}\left\|p_{1}-p_{2}\right\|_{L^{r}}, \quad \text { and } \quad b\left(\mathbf{u}_{1}-\mathbf{u}_{2}, q\right) \leq\left\|\nabla \cdot\left(\mathbf{u}_{1}-\mathbf{u}_{2}\right)\right\|_{L^{*}}\|q\|_{L^{r}} .
\end{aligned}
$$

From (2.4), we find that

$$
\begin{aligned}
\left(\left|\nabla \cdot \mathbf{u}_{1}\right|^{r^{*}-2} \nabla \cdot \mathbf{u}_{1}-\left|\nabla \cdot \mathbf{u}_{2}\right|^{r^{*}-2} \nabla \cdot \mathbf{u}_{2}, \nabla \cdot \mathbf{v}\right) & \leq C\left(\left.|1+| \nabla \cdot \mathbf{u}_{1}\right|^{r^{*}-2}+\left|\nabla \cdot \mathbf{u}_{2}\right|^{r^{*}-2}|\cdot| \nabla \cdot\left(\mathbf{u}_{1}-\mathbf{u}_{2}\right)|,| \nabla \cdot \mathbf{v} \mid\right) \\
& \leq C\left(1+\left\|\nabla \cdot \mathbf{u}_{1}\right\|_{L^{*}}^{r^{*}-2}+\left\|\nabla \cdot \mathbf{u}_{2}\right\|_{L^{*}}^{*^{*}-2}\right)\left\|\nabla \cdot\left(\mathbf{u}_{1}-\mathbf{u}_{2}\right)\right\|_{L^{*}}\|\nabla \cdot \mathbf{v}\|_{L^{*}}
\end{aligned}
$$


From the above it follows that

$$
\begin{aligned}
\left\langle\mathcal { A } _ { \varepsilon } \left(\left(\mathbf{u}_{1}, p_{1}\right)-\right.\right. & \left.\mathcal{A}_{\varepsilon}\left(\left(\mathbf{u}_{2}, p_{2}\right),(\mathbf{v}, q)\right)\right\rangle_{(V \times Q)^{\prime} \times(V \times Q)} \leq C_{\varepsilon}\left(1+\left\|\mathbf{u}_{1}\right\|_{L^{s}}^{s-2}+\left\|\mathbf{u}_{2}\right\|_{L^{s}}^{s-2}\right) \\
& \left(1+\left\|\nabla \cdot \mathbf{u}_{1}\right\|_{L^{*}}^{r^{*}-2}+\left\|\nabla \cdot \mathbf{u}_{2}\right\|_{L^{*}}^{r^{*}-2}\right)\left(\left\|\mathbf{u}_{1}-\mathbf{u}_{2}\right\|_{V}+\left\|p_{1}-p_{2}\right\|_{Q}+\left\|p_{1}-p_{2}\right\|_{Q}^{\lambda}\right)\left(\|\mathbf{v}\|_{V}+\|q\|_{Q}\right),
\end{aligned}
$$

for all $\mathbf{v} \in V, q \in Q$. This yields

$$
\begin{aligned}
& \| \mathcal{A}_{\varepsilon}\left(\left(\mathbf{u}_{1}, p_{1}\right)-\mathcal{A}_{\varepsilon}\left(\left(\mathbf{u}_{2}, p_{2}\right) \|_{(V \times Q)^{\prime}}\right.\right. \leq C_{\varepsilon}\left(1+\left\|\mathbf{u}_{1}\right\|_{L^{s}}^{S-2}+\left\|\mathbf{u}_{2}\right\|_{L^{s}}^{S-2}\right) \\
&\left(1+\left\|\nabla \cdot \mathbf{u}_{1}\right\|_{L^{r^{*}}}^{r^{*}-2}+\left\|\nabla \cdot \mathbf{u}_{2}\right\| \|_{L^{*}}^{r^{*}-2}\right)\left(\left\|\mathbf{u}_{1}-\mathbf{u}_{2}\right\|_{V}+\left\|p_{1}-p_{2}\right\|_{Q}+\left\|p_{1}-p_{2}\right\|_{Q}^{\lambda}\right) .
\end{aligned}
$$

For $\mathcal{A}_{\varepsilon}$ is the coercive.

$$
\begin{aligned}
\left\langle\mathcal{A}_{\varepsilon}(\mathbf{u}, p),(\mathbf{u}, p)\right\rangle_{(V \times Q)^{\prime} \times(V \times Q)} & \geq C\left(\|\mathbf{u}\|^{2}+\|\mathbf{u}\|_{L^{s}}^{s}\right)+\varepsilon\left(\|\nabla \cdot \mathbf{u}\|_{L^{L^{*}}}^{r^{*}}+\|p\|_{Q}^{r}\right) \\
& \geq C_{\varepsilon}\left(\|\mathbf{u}\|_{L^{s}}^{s}+\|\nabla \cdot \mathbf{u}\|_{L^{r^{*}}}^{r^{*}}+\|p\|_{Q}^{r}\right),
\end{aligned}
$$

whence

$$
\frac{\left\langle\mathcal{A}_{\varepsilon}(\mathbf{u}, p),(\mathbf{u}, p)\right\rangle_{(V \times Q)^{\prime} \times(V \times Q)}}{\|\mathbf{u}\|_{V}+\|p\|_{Q}} \geq C_{\varepsilon} \frac{\|\mathbf{u}\|_{L^{s}}^{s}+\|\nabla \cdot \mathbf{u}\|_{L^{*}}^{r^{*}}+\|p\|_{Q}^{r}}{\|\mathbf{u}\|_{L^{s}}+\|\nabla \cdot \mathbf{u}\|_{L^{L^{*}}}+\|p\|_{Q}} .
$$

Therefore we deduce that

$$
\lim _{\|(\mathbf{v}, q)\|_{V \times Q} \rightarrow+\infty} \frac{\left\langle\mathcal{A}_{\varepsilon}(\mathbf{u}, p),(\mathbf{u}, p)\right\rangle_{(V \times Q)^{\prime} \times(V \times Q)}}{\|\mathbf{u}\|_{V}+\|p\|_{Q}}=+\infty .
$$

For $\mathcal{A}_{\varepsilon}$ is the strictly monotone.

$$
\begin{aligned}
&\left\langle\mathcal{A}_{\varepsilon}(\mathbf{u}, p)-\mathcal{A}_{\varepsilon}(\mathbf{v}, q),(\mathbf{u}-\mathbf{v}, p-q)\right\rangle_{(V \times Q)^{\prime} \times(V \times Q)} \\
& \geq C\left(\|\mathbf{u}-\mathbf{v}\|^{2}+\|\mathbf{u}-\mathbf{v}\|_{L^{s}}^{s}\right)+\varepsilon\left(\|\nabla \cdot(\mathbf{u}-\mathbf{v})\|_{L^{r^{*}}}^{r^{*}}+\|p-q\|_{Q}^{r}\right) \\
& \quad \geq C_{\varepsilon}\left(\|\mathbf{u}-\mathbf{v}\|_{L^{s}}^{s}+\|\nabla \cdot(\mathbf{u}-\mathbf{v})\|_{L^{*}}^{r^{*}}+\|p-q\|_{Q}^{r}\right)>0 \quad \text { for all }(\mathbf{u}, p) \neq(\mathbf{v}, q) .
\end{aligned}
$$

Step 2. Next, we show that the solution $\left(\mathbf{m}_{\varepsilon}, u_{\varepsilon}\right)$ is bounded independently of $\varepsilon$. To do this, we use the following result (see in [15] Lemma A.3 or [22] Lemma A.1),

Lemma 3.3. Let $s>1$ and $1 / s+1 / s^{*}=1$. Then there exists a constant $C_{*}>0$ such that

$$
C_{*}\|q\|_{L^{s^{*}}} \leq \sup _{\mathbf{v} \in W(\operatorname{div}, \Omega)} \frac{b(\mathbf{v}, q)}{\|\mathbf{v}\|_{W(\operatorname{div}, \Omega)}} \quad \text { for all } \mathbf{v} \in W(\operatorname{div}, \Omega), \mathrm{q} \in \mathrm{L}^{\mathrm{s}}(\Omega) .
$$

Lemma 3.4. There exists $C>0$ independent of $\varepsilon$ such that for sufficiently small $\varepsilon>0$ the solution $\left(\mathbf{m}_{\varepsilon}, u_{\varepsilon}\right)$ of (3.4) satisfies the following estimates

$$
\left\|u_{\varepsilon}\right\|_{Q}+\left\|\mathbf{m}_{\varepsilon}\right\|_{V} \leq C
$$


Proof. We begin with a bound for the norm of $\nabla \cdot \mathbf{m}_{\varepsilon}$. Using the second equation of (3.4) with $q=\operatorname{sgn}\left(\nabla \cdot \mathbf{m}_{\varepsilon}\right)\left|\nabla \cdot \mathbf{m}_{\varepsilon}\right|^{r^{*}-1}$, we obtain

$$
\left\|\nabla \cdot \mathbf{m}_{\varepsilon}\right\|_{L^{r^{*}}}^{r^{*}} \leq\|f\|_{L^{r^{*}}}\left\|\nabla \cdot \mathbf{m}_{\varepsilon}\right\|_{L^{*}}^{r^{*}-1}+\varepsilon\left\|u_{\varepsilon}\right\|_{L^{r}}^{\lambda}\left\|\nabla \cdot \mathbf{m}_{\varepsilon}\right\|_{L^{r^{*}}}^{r^{*}-1}
$$

It implies that

$$
\left\|\nabla \cdot \mathbf{m}_{\varepsilon}\right\|_{L^{r^{*}}} \leq\|f\|_{L^{r^{*}}}+\varepsilon\left\|u_{\varepsilon}\right\|_{Q}^{\lambda}
$$

Taking the test function $(\mathbf{v}, q)=\left(\mathbf{m}_{\varepsilon}, u_{\varepsilon}\right)$ in (3.4) gives

$$
\begin{aligned}
a\left(\mathbf{m}_{\varepsilon},\right. & \left.\mathbf{m}_{\varepsilon}\right)+\varepsilon\left(\left|\nabla \cdot \mathbf{m}_{\varepsilon}\right|^{r^{*}-2} \nabla \cdot \mathbf{m}_{\varepsilon}, \nabla \cdot \mathbf{m}_{\varepsilon}\right)+\varepsilon\left(u_{\varepsilon}^{\lambda}, u_{\varepsilon}\right) \\
\quad & =-\left\langle u_{b}, \mathbf{m}_{\varepsilon} \cdot v\right\rangle+\left(f, u_{\varepsilon}\right)=-\left(\nabla \cdot \mathbf{m}_{\varepsilon}, u_{b}\right)-\left(\nabla u_{b}, \mathbf{m}_{\varepsilon}\right)+\left(f, u_{\varepsilon}\right) \\
& \leq\left\|u_{b}\right\|_{V^{\prime}}\left(\left\|\mathbf{m}_{\varepsilon}\right\|_{L^{s}}+\left\|\nabla \cdot \mathbf{m}_{\varepsilon}\right\|_{L^{*}}\right)+\|f\|_{L^{*}}\left\|u_{\varepsilon}\right\|_{L^{r}} .
\end{aligned}
$$

Using (3.9) and the fact that $a\left(\mathbf{m}_{\varepsilon}, \mathbf{m}_{\varepsilon}\right) \geq C\left(\left\|\mathbf{m}_{\varepsilon}\right\|_{L^{s}}^{s}+\left\|\mathbf{m}_{\varepsilon}\right\|_{L^{2}}^{2}\right)$, we may conclude that

$$
C\left\|\mathbf{m}_{\varepsilon}\right\|_{L^{s}}^{S}+\varepsilon\left(\left\|\nabla \cdot \mathbf{m}_{\varepsilon}\right\|_{L^{r^{*}}}^{r^{*}}+\left\|u_{\varepsilon}\right\|_{Q}^{r}\right) \leq\left\|u_{b}\right\|_{V^{\prime}}\left(\left\|\mathbf{m}_{\varepsilon}\right\|_{L^{s}}+\|f\|_{L^{r^{*}}}+\varepsilon\left\|u_{\varepsilon}\right\|_{Q}^{\lambda}\right)+\|f\|_{L^{L^{*}}}\left\|u_{\varepsilon}\right\|_{Q}
$$

To bound $u_{\varepsilon}$ we employ the inf-sup condition (3.7). The first equation of (3.4) and the above estimate for $\left\|\nabla \cdot \mathbf{m}_{\varepsilon}\right\|_{L^{r^{*}}}$, we have

$$
\begin{aligned}
& C_{*}\left\|u_{\varepsilon}\right\|_{Q} \leq \sup _{\mathbf{v} \in V} \frac{b\left(\mathbf{v}, u_{\varepsilon}\right)}{\|\mathbf{v}\|_{V}}=\sup _{\mathbf{v} \in V} \frac{a\left(\mathbf{m}_{\varepsilon}, \mathbf{v}\right)+\varepsilon\left(\nabla \cdot \mathbf{m}_{\varepsilon} r^{r^{*}-2} \nabla \cdot \mathbf{m}_{\varepsilon}, \nabla \cdot \mathbf{v}\right)+\left\langle u_{b}, \mathbf{v} \cdot v\right\rangle}{\|\mathbf{v}\|_{V}} \\
& \quad \leq \sup _{\mathbf{v} \in V} \frac{C\left(\left\|\mathbf{m}_{\varepsilon}\right\|_{L^{s}}+\left\|\mathbf{m}_{\varepsilon}\right\|_{L^{s}}^{s-1}\right)\|\mathbf{v}\|_{L^{s}}+\varepsilon\left\|\nabla \cdot \mathbf{m}_{\varepsilon}\right\|_{L^{r^{*}}}^{r^{*}}\|\nabla \cdot \mathbf{v}\|_{L^{r^{*}}}+\left\|u_{b}\right\|_{V^{\prime}}\left(\|\mathbf{v}\|_{L^{s}}+\|\nabla \cdot \mathbf{v}\|\right)}{\|\mathbf{v}\|_{V}} \\
& \quad \leq C\left(\left\|\mathbf{m}_{\varepsilon}\right\|_{L^{s}}+\left\|\mathbf{m}_{\varepsilon}\right\|_{L^{s}}^{s-1}\right)+\varepsilon\left(\|f\|_{L^{r^{*}}}+\varepsilon\left\|u_{\varepsilon}\right\|_{Q}^{\lambda}\right)^{r^{*}-1}+\left\|u_{b}\right\|_{V^{\prime}} \\
& \quad \leq C\left(\left\|\mathbf{m}_{\varepsilon}\right\|_{L^{s}}+\left\|\mathbf{m}_{\varepsilon}\right\|_{L^{s}}^{s-1}\right)+\varepsilon 2^{r^{*}-2}\|f\|_{L^{*}}^{r^{*}-1}+\varepsilon^{2} 2^{r^{*}-2}\left\|u_{\varepsilon}\right\|_{Q}+\left\|u_{b}\right\|_{V^{\prime}}
\end{aligned}
$$

for some constant $C_{*}>0$. Hence, for sufficiently small $\varepsilon$ (e.g., $\left.\varepsilon \leq\left(2^{1-r^{*}} C_{*}\right)^{1 / 2}\right)$,

$$
\left\|u_{\varepsilon}\right\|_{Q} \leq C\left(\left\|\mathbf{m}_{\varepsilon}\right\|_{L^{s}}+\left\|\mathbf{m}_{\varepsilon}\right\|_{L^{s}}^{S-1}+\|f\|_{L^{r^{*}}}^{r^{*}-1}+\left\|u_{b}\right\|_{V^{\prime}}\right)
$$

Substituting (3.12) into (3.11) leads to

$$
\left\|\mathbf{m}_{\varepsilon}\right\|_{L^{s}}^{s} \leq C\left\|u_{b}\right\|_{V^{\prime}}\left(\left\|\mathbf{m}_{\varepsilon}\right\|_{L^{s}}+\|f\|_{L^{*}}\right)+C\left(\left\|u_{b}\right\|_{V^{\prime}}+\|f\|_{L^{r^{*}}}\right)\left(\left\|\mathbf{m}_{\varepsilon}\right\|_{L^{s}}^{s-1}+\left\|\mathbf{m}_{\varepsilon}\right\|_{L^{s}}+\|f\|_{L^{*}}^{r^{*}}-1+\left\|u_{b}\right\|_{V^{\prime}}+1\right) .
$$

Then by using Young's inequality, we obtain

$$
\left\|\mathbf{m}_{\varepsilon}\right\|_{L^{s}}^{s} \leq C_{1},
$$

where $C_{1}=C\left(\left\|u_{b}\right\|_{V^{\prime}}+\|f\|_{L^{r^{*}}}\right)\left(\left\|u_{b}\right\|_{V^{\prime}}+\|f\|_{L^{r^{*}}}^{r^{*}-1}\right)+\left(\left\|u_{b}\right\|_{V^{\prime}}+\|f\|_{L^{r^{*}}}\right)^{s}+1$.

Insert (3.13) into (3.12) yields

$$
\left\|u_{\varepsilon}\right\|_{Q} \leq C_{2}
$$

where $C_{2}=C_{1}^{1 / s}+C_{1}^{(s-1) / s}+\|f\|_{L^{*}}^{r^{*}-1}+\left\|u_{b}\right\|_{V^{\prime}}$. Using this estimate in (3.9) yields

$$
\left\|\nabla \cdot \mathbf{m}_{\varepsilon}\right\|_{L^{r^{*}}} \leq\|f\|_{L^{r^{*}}}+C_{2}^{r^{*}-1}
$$


Therefore

$$
\left\|\mathbf{m}_{\varepsilon}\right\|_{V} \leq C_{1}+C_{2}^{r^{*}-1} .
$$

The assertion of the lemma follows from (3.14) and (3.15). $\mathrm{C}$

Step 3. Adding the left hand side of (3.2), we obtain the following nonlinear form defined on $V \times Q$ by

$$
a((\mathbf{m}, u),(\mathbf{v}, q)):=a(\mathbf{m}, \mathbf{v})-b(\mathbf{v}, u)+b(\mathbf{m}, q) .
$$

Consider the nonlinear operator $\mathcal{A}: V \times Q \rightarrow(V \times Q)^{\prime}$ defined by

$$
\langle\mathcal{A}(\mathbf{u}, p),(\mathbf{v}, q)\rangle_{(V \times Q)^{\prime} \times(V \times Q)}:=a((\mathbf{u}, p),(\mathbf{v}, q)) .
$$

Set $\varepsilon=1 / n$, and let $\left(\mathbf{m}_{n}, u_{n}\right)$ be the unique solution of the regularized problem (3.4). Since $\left(\mathbf{m}_{n}, u_{n}\right)$ is a bounded sequence in $V \times Q$, there exists a weakly convergent subsequence, again denoted by $\left(\mathbf{m}_{n}, u_{n}\right)$, with weak limit $(\mathbf{m}, u) \in V \times Q$. For $\tilde{f}(\mathbf{v}, q):=-\left\langle u_{b}, \mathbf{v} \cdot v\right\rangle+(f, q) \in(V \times Q)^{\prime}$,

$$
\begin{aligned}
\left\|\mathcal{A}\left(\mathbf{m}_{n}, u_{n}\right)-\tilde{f}\right\|_{(V \times Q)^{\prime}} & =\sup _{(\mathbf{v}, q) \neq \mathbf{0}} \frac{\left|a\left(\left(\mathbf{m}_{n}, u_{n}\right),(\mathbf{v}, q)\right)-\tilde{f}(\mathbf{v}, q)\right|}{\|(\mathbf{v}, q)\|_{V \times Q}} \\
& =\sup _{(\mathbf{v}, q) \neq \mathbf{0}} \frac{\left|a\left(\mathbf{m}_{n}, \mathbf{v}\right)-b\left(\mathbf{v}, u_{n}\right)+b\left(\mathbf{m}_{n}, q\right)-\tilde{f}(\mathbf{v}, q)\right|}{\|(\mathbf{v}, q)\|_{V \times Q}} .
\end{aligned}
$$

Noting from (3.4) that

$$
\begin{aligned}
\left|a\left(\mathbf{m}_{n}, \mathbf{v}\right)-b\left(\mathbf{v}, u_{n}\right)+b\left(\mathbf{m}_{n}, q\right)-\tilde{f}(\mathbf{v}, q)\right| & =\frac{1}{n}\left|\left(\left|\nabla \cdot \mathbf{m}_{n}\right|^{r^{*}-2} \nabla \cdot \mathbf{m}_{n}, \nabla \cdot \mathbf{v}\right)+\left(u_{n}^{\lambda}, q\right)\right| \\
& \leq \frac{C}{n}\left(\left\|\nabla \cdot \mathbf{m}_{n}\right\|_{L^{*}}^{*^{*}-1}\|\nabla \cdot \mathbf{v}\|_{L^{r^{*}}}+\left\|u_{n}\right\|_{L^{r}}\|q\|_{L^{r}}\right) \\
& \leq \frac{C}{n}\left(\left\|\nabla \cdot \mathbf{m}_{n}\right\|_{L^{r^{*}}}^{*-1}+\left\|u_{n}\right\|_{Q}\right)\|(\mathbf{v}, q)\|_{V \times Q} .
\end{aligned}
$$

Hence

$$
\left\|\mathcal{A}\left(\mathbf{m}_{n}, u_{n}\right)-\tilde{f}\right\|_{(V \times Q)^{\prime}} \leq \frac{C}{n}\left(\left\|\nabla \cdot \mathbf{m}_{n}\right\|_{L^{r^{*}}}^{r^{*}-1}+\left\|u_{n}\right\|_{Q}\right) \stackrel{n \rightarrow \infty}{\longrightarrow} 0 .
$$

The sequence $\mathcal{A}\left(\mathbf{m}_{n}, u_{n}\right)$ converges strongly in $(V \times Q)^{\prime}$ to $\tilde{f}$. Thus we can conclude that $\mathcal{A}(\mathbf{m}, u)=\tilde{f}$ in $(V \times Q)^{\prime}$ (see e.g. [25], p. 474), i.e., $(\mathbf{m}, u)$ is a solution of problem (3.3).

Step 4. To show the uniqueness we consider two solutions $\left(\mathbf{m}_{1}, u_{1}\right)$ and $\left(\mathbf{m}_{2}, u_{2}\right)$ of $(3.2)$. Using the test function $(\mathbf{v}, q)=\left(\mathbf{m}_{1}-\mathbf{m}_{2}, u_{1}-u_{2}\right)$, we obtain

$$
\begin{array}{r}
a\left(\mathbf{m}_{1}, \mathbf{m}_{1}-\mathbf{m}_{2}\right)-a\left(\mathbf{m}_{2}, \mathbf{m}_{1}-\mathbf{m}_{2}\right)-\left(b\left(\mathbf{m}_{1}-\mathbf{m}_{2}, u_{1}\right)-b\left(\mathbf{m}_{1}-\mathbf{m}_{2}, u_{2}\right)\right)=0, \\
b\left(\mathbf{m}_{1}, u_{1}-u_{2}\right)-b\left(\mathbf{m}_{2}, u_{1}-u_{2}\right)=0 .
\end{array}
$$

Adding these equations and using the monotonicity of $F(\cdot)$ in $(2.5)$ yield

$$
0=a\left(\mathbf{m}_{1}, \mathbf{m}_{1}-\mathbf{m}_{2}\right)-a\left(\mathbf{m}_{2}, \mathbf{m}_{1}-\mathbf{m}_{2}\right) \geq C_{2}\left(\left\|\mathbf{m}_{1}-\mathbf{m}_{2}\right\|_{L^{2}}^{2}+\left\|\mathbf{m}_{1}-\mathbf{m}_{2}\right\|_{L^{s}}^{s}\right) .
$$

It follows that $\mathbf{m}_{1}=\mathbf{m}_{2}$. If $\mathbf{m} \in V$ is given then $u \in L^{r}(\Omega)$ is defined as a solution of the variational equation $b(\mathbf{v}, u)=\left\langle u_{b}, \mathbf{v} \cdot v\right\rangle+a(\mathbf{m}, \mathbf{v})$ for all $\mathbf{v} \in V$. The uniqueness of $u$ is directly consequence of Lemma $3.3 \mathbf{Z}$ 
4. The semi-discrete problem. We return to the transient problem governed by (1.13). We discretize (1.13) in time using the implicit Euler method. This yields not only a method to solve the transient problem numerically, but also an approach to prove its solvability, the technique of semi-discretization. We define a partition $0=t_{0}<t_{1}<\ldots<t_{J}=T$ of the segment $[0, T]$ into $J$ intervals of constant length $h=T / J$, i.e., $t_{j}=j h$ for $j=0, \ldots, J$. In the following for $j=0, \ldots, J$ we use the denotations $u^{j}:=u(\cdot, j t)$ and $\mathbf{m}^{j}:=\mathbf{m}(\cdot, j t)$ for the unknown solutions and, analogously defined, $u_{b}^{j}$ for the boundary conditions and $f^{j}$ for the source term.

$$
\begin{array}{rl}
\left(\sum_{i=0}^{N} a_{i}^{j}\left|\mathbf{m}^{j}\right|^{\alpha_{i}}\right) \mathbf{m}^{j} & =-\nabla u^{j} \quad x \in \Omega, \\
\phi \frac{\left(u^{j}\right)^{\lambda}-\left(u^{j-1}\right)^{\lambda}}{h}+\nabla \cdot \mathbf{m}^{j}=f^{j} & x \in \Omega, \\
u & =-u_{b}^{j} \quad x \in \partial \Omega, \\
u(x, 0) & =u_{0}(x) \quad x \in \Omega .
\end{array}
$$

For each $j \in\{1, \ldots, J\}$, we will make the following assumptions:

$$
0<\underline{\phi} \leq \phi(x) \leq \bar{\phi}<\infty ; f^{j} \in L^{r^{*}}(\Omega) ; u_{b}^{j} \in W^{1 / s, s}(\partial \Omega), u_{0} \in W^{1, s^{*}}(\Omega) \cap L^{r}(\Omega) ; a_{i}^{j}(x) \in L^{\infty}(\Omega), i=0, \ldots, N .
$$

Mixed formulation of the semi-discrete problem. The discretization in time of the continuity equation (4.1) with the implicit Euler method yields for each $j \in\{1, \ldots, J\}$. Find $\left(\mathbf{m}^{j}, u^{j}\right) \in V \times Q$ such that

$$
\begin{array}{r}
\left(\left(\sum_{i=0}^{N} a_{i}^{j}\left|\mathbf{m}^{j}\right|^{\alpha_{i}}\right) \mathbf{m}^{j}, \mathbf{v}\right)-\left(u^{j}, \nabla \cdot \mathbf{v}\right)=-\left\langle u_{b}^{j}, \mathbf{v} \cdot v\right\rangle \quad \text { for all } \mathbf{v} \in V, \\
\left(\phi \frac{\left(u^{j}\right)^{\lambda}}{h}, q\right)+\left(\nabla \cdot \mathbf{m}^{j}, q\right)=\left(f^{j}, q\right)+\left(\phi \frac{\left(u^{j-1}\right)^{\lambda}}{h}, q\right) \quad \text { for all } q \in Q,
\end{array}
$$

with $u^{0}=u_{0}(x)$. Using $a$ and $b$ are defined in Section 3, we rewrite the mixed formulation (4.2) in the following way: Find $\left(\mathbf{m}^{j}, u^{j}\right) \in V \times Q$, such that

$$
\begin{array}{ll}
a\left(\mathbf{m}^{j}, \mathbf{v}\right)-b\left(\mathbf{v}, u^{j}\right)=-\left\langle u_{b}^{j}, \mathbf{v} \cdot v\right\rangle & \text { for all } \mathbf{v} \in V, \\
\left(\phi \frac{\left(u^{j}\right)^{\lambda}}{h}, q\right)+b\left(\mathbf{m}^{j}, q\right)=\left(\bar{f}^{j}, q\right) & \text { for all } q \in Q,
\end{array}
$$

where $\bar{f}^{j}=f^{j}+\frac{\phi}{h}\left(u^{j-1}\right)^{\lambda}$.

The remainder of this section we restrict our considerations to the problem (4.3) for a fixed time step $j$. For simplicity, we omit the superscript $j$.

4.1. Regularization of the semi-discrete problem. We use the technique of regularization again. For the fixed $\varepsilon>0$, we consider the following regularized problem. Find $\left(\mathbf{m}_{\varepsilon}, u_{\varepsilon}\right) \in V \times Q$ such that

$$
\begin{aligned}
a\left(\mathbf{m}_{\varepsilon}, \mathbf{v}\right)+\varepsilon\left(\left|\nabla \cdot \mathbf{m}_{\varepsilon}\right|^{*^{*}-2} \nabla \cdot \mathbf{m}_{\varepsilon}, \nabla \cdot \mathbf{v}\right)-b\left(\mathbf{v}, u_{\varepsilon}\right)=-\left\langle u_{b}, \mathbf{v} \cdot v\right\rangle & \text { for all } \mathbf{v} \in V, \\
\left(\frac{\phi}{h} u_{\varepsilon}^{\lambda}, q\right)+b\left(\mathbf{m}_{\varepsilon}, q\right)=(\bar{f}, q) & \text { for all } q \in Q .
\end{aligned}
$$

The following result may be proved in much the same manner as Lemma 3.2 
Lemma 4.1. For every $\varepsilon$, there exists a unique solution $\left(\mathbf{m}_{\varepsilon}, u_{\varepsilon}\right) \in V \times Q$ of the regularized semidiscrete problem (4.4).

Next, we show that the solution $\left(\mathbf{m}_{\varepsilon}, u_{\varepsilon}\right)$ of (4.4) is bounded independently of $\varepsilon$.

Lemma 4.2. There exists $C>0$ independent of $\varepsilon$ such that for sufficiently small $\varepsilon>0$ the solution $\left(\mathbf{m}_{\varepsilon}, u_{\varepsilon}\right)$ of (4.4) satisfies

$$
\left\|u_{\varepsilon}\right\|_{Q}+\left\|\mathbf{m}_{\varepsilon}\right\|_{V} \leq C
$$

Proof. As in the proof of Lemma 3.4, we begin with an estimate for the norm of $\nabla \cdot \mathbf{m}_{\varepsilon}$. Using the second equation of (4.4) with $q=\operatorname{sgn}\left(\nabla \cdot \mathbf{m}_{\varepsilon}\right) \mid \nabla \cdot \mathbf{m}_{\varepsilon} r^{r^{*}-1}$, we obtain

$$
\left\|\nabla \cdot \mathbf{m}_{\varepsilon}\right\|_{L^{*}} \leq\|\bar{f}\|_{L^{r^{*}}}+\frac{\bar{\phi}}{h}\left\|u_{\varepsilon}\right\|_{Q}^{\lambda} .
$$

The estimation of $\left\|\mathbf{m}_{\varepsilon}\right\|_{L^{s}}$ is based on choosing the test function $(\mathbf{v}, q)=\left(\mathbf{m}_{\varepsilon}, u_{\varepsilon}\right)$ in (4.4). Then we obtain the estimate

$$
a\left(\mathbf{m}_{\varepsilon}, \mathbf{m}_{\varepsilon}\right)+\varepsilon\left(\left|\nabla \cdot \mathbf{m}_{\varepsilon}\right|^{r^{*}-2} \nabla \cdot \mathbf{m}_{\varepsilon}, \nabla \cdot \mathbf{m}_{\varepsilon}\right)+\left(\phi \frac{u_{\varepsilon}^{\lambda}}{h}, u_{\varepsilon}\right) \leq\left\|u_{b}\right\|_{V^{\prime}}\left(\left\|\mathbf{m}_{\varepsilon}\right\|_{L^{s}}+\left\|\nabla \cdot \mathbf{m}_{\varepsilon}\right\|_{L^{r^{*}}}\right)+\|f\|_{L^{*}}\left\|u_{\varepsilon}\right\|_{Q} .
$$

Thanks to the monotonicity of the function $F(\cdot)$ and (4.6), it follows from (4.7) that

$$
C\left(\left\|\mathbf{m}_{\varepsilon}\right\|_{L^{s}}^{s}+\left\|\mathbf{m}_{\varepsilon}\right\|^{2}\right)+\varepsilon\left\|\nabla \cdot \mathbf{m}_{\varepsilon}\right\|_{L^{r^{*}}}+\frac{\phi}{\bar{h}}\left\|u_{\varepsilon}\right\|_{Q}^{r} \leq\left\|u_{b}\right\|_{V^{\prime}}\left(\left\|\mathbf{m}_{\varepsilon}\right\|_{L^{s}}+\|\bar{f}\|_{L^{r^{*}}}+\frac{\bar{\phi}}{h}\left\|u_{\varepsilon}\right\|_{Q}^{\lambda}\right)+\|\bar{f}\|_{L^{L^{*}}}\left\|u_{\varepsilon}\right\|_{Q} .
$$

This and Young's inequality show that

$$
\left\|\mathbf{m}_{\varepsilon}\right\|_{L^{s}}^{s}+\frac{\phi}{2 h}\left\|u_{\varepsilon}\right\|_{Q}^{r} \leq C\left(\left\|u_{b}\right\|_{V^{\prime}}^{s^{*}}+\left\|u_{b}\right\|_{V^{\prime}}\|\bar{f}\|_{L^{r^{*}}}+\left\|u_{b}\right\|_{V^{\prime}}^{r}+\|\bar{f}\|_{L^{r^{*}}}^{r^{*}}\right)
$$

which leads to

$$
\left\|\mathbf{m}_{\varepsilon}\right\|_{L^{s}} \leq C\left(\left\|u_{b}\right\|_{V^{\prime}}^{s^{*}}+\left\|u_{b}\right\|_{V^{\prime}}^{r}+\|\bar{f}\|_{L^{r^{*}}}^{r^{*}}\right)^{1 / s}, \quad\left\|u_{\varepsilon}\right\|_{Q} \leq C\left(\left\|u_{b}\right\|_{V^{\prime}}^{s^{*}}+\left\|u_{b}\right\|_{V^{\prime}}^{r}+\|\bar{f}\|_{L^{*}}^{r^{*}}\right)^{1 / r} .
$$

Substituting (4.8) into (4.6) we can assert that

$$
\begin{aligned}
\left\|\nabla \cdot \mathbf{m}_{\varepsilon}\right\|_{L^{*}} & \leq\|\hat{f}\|_{L^{r^{*}}}+C\left(\left\|u_{b}\right\|_{V^{\prime}}^{s^{*}}+\left\|u_{b}\right\|_{V^{\prime}}^{r}+\|\vec{f}\|_{L^{r^{*}}}^{r^{*}}\right)^{\lambda / r} \\
& \leq C\left(1+\left\|u_{b}\right\|_{V^{\prime}}^{s^{*} / r^{*}}+\left\|u_{b}\right\|_{V^{\prime}}^{\lambda}+\|\bar{f}\|_{L^{*^{*}}}\right) .
\end{aligned}
$$

The assertion (4.5) follows directly from (4.8)-(4.9).

4.2. Solvability of the semi-discrete problem. In the same manner as in Section 1, we pass the limit $\varepsilon \rightarrow 0$ and obtain the existence of a solution of the semi-discrete problem (4.2).

THEOREM 4.3. The mixed formulation (4.2) of the semi-discrete problem (4.1) possesses a unique solution $(\mathbf{m}, u) \in W(\operatorname{div} ; \Omega) \times \mathrm{L}^{\mathrm{r}}(\Omega)$.

Proof. Analysis similar to that in the proof of Theorem 3.1, we add two equations in (4.3) and obtain the nonlinear form $a$, defined on $(V \times Q) \times(V \times Q)^{\prime}$, and the linear form $\tilde{f} \in(V \times Q)^{\prime}$, defined by

$$
a((\mathbf{m}, u),(\mathbf{v}, q)) \stackrel{\text { def }}{=} a(\mathbf{m}, \mathbf{v})-b(\mathbf{v}, u)+\left(\frac{\phi u}{h}, q\right)+b(\mathbf{m}, q), \quad \tilde{f}(\mathbf{v}, q) \stackrel{\text { def }}{=}-\left\langle u_{b}, \mathbf{v} \cdot v\right\rangle+(\bar{f}, q) .
$$


Again, the operator $\mathcal{A}: V \times Q \rightarrow(V \times Q)^{\prime}$ is defined by

$$
\langle\mathcal{A}(\mathbf{u}, p),(\mathbf{v}, q)\rangle_{(V \times Q)^{\prime} \times(V \times Q)}=a((\mathbf{u}, p),(\mathbf{v}, q)) .
$$

Choosing $\varepsilon=1 / n$, we obtain a sequence of unique solutions $\left(\mathbf{m}_{n}, u_{n}\right)$ of the regularized problems (4.4). Owing to Lemma 4.2 the sequence $\left(\left(\mathbf{m}_{n}, u_{n}\right)\right)_{n \in \mathbb{N}}$ is bounded in $V \times Q$. Hence there is a weakly convergent subsequence, again denoted by $\left(\left(\mathbf{m}_{n}, u_{n}\right)\right)_{n \in \mathbb{N}}$, which converges to $(\mathbf{m}, \rho) \in V \times Q$. In the same manner as in the proof of Theorem 3.1 we obtain the identity $\mathcal{A}(\mathbf{m}, \rho)=\tilde{f}$ in $(V \times Q)^{\prime}$, i.e., $(\mathbf{m}, \rho)$ is a solution of the semi-discrete mixed formulation (4.2).

To show the uniqueness, we consider two solutions $\left(\mathbf{m}_{1}, u_{1}\right)$ and $\left(\mathbf{m}_{2}, u_{2}\right)$ of (4.3). Using the test function $(\mathbf{v}, q)=\left(\mathbf{m}_{1}-\mathbf{m}_{2}, u_{1}-u_{2}\right)$, we obtain

$$
\begin{array}{r}
a\left(\mathbf{m}_{1}, \mathbf{m}_{1}-\mathbf{m}_{2}\right)-a\left(\mathbf{m}_{2}, \mathbf{m}_{1}-\mathbf{m}_{2}\right)-b\left(\mathbf{m}_{1}-\mathbf{m}_{2}, u_{1}-u_{2}\right)=0 \\
\left(\phi \frac{u_{1}^{\lambda}-u_{2}^{\lambda}}{h}, u_{1}-u_{2}\right)+b\left(\mathbf{m}_{1}-\mathbf{m}_{2}, u_{1}-u_{2}\right)=0 .
\end{array}
$$

Adding the two equations then using (2.5) and (2.8) yields

$$
\begin{aligned}
0 & =a\left(\mathbf{m}_{1}, \mathbf{m}_{1}-\mathbf{m}_{2}\right)-a\left(\mathbf{m}_{2}, \mathbf{m}_{1}-\mathbf{m}_{2}\right)+\left(\phi \frac{u_{1}^{\lambda}-u_{2}^{\lambda}}{h}, u_{1}-u_{2}\right) \\
& \geq C_{2}\left(\left\|\mathbf{m}_{1}-\mathbf{m}_{2}\right\|^{2}+\left\|\mathbf{m}_{1}-\mathbf{m}_{2}\right\|_{L^{s}}^{s}\right)+\int_{\Omega} \frac{\phi}{h} \frac{\left|u_{1}-u_{2}\right|^{2}}{\left|u_{1}\right|^{1-\lambda}+\left|u_{2}\right|^{1-\lambda}} d x,
\end{aligned}
$$

which proves $\mathbf{m}_{1}=\mathbf{m}_{2}$ and $u_{1}=u_{2}$ a.e.

5. The transient problem. We address the continuous transient problem. Due to the lack of regularity of the solution $\mathbf{m}$, it is impossible to handle more general boundary conditions as in the previous sections. We will restrict our considerations here to the case of homogeneous Dirichlet boundary conditions

$$
\begin{array}{rr}
\left(\sum_{i=0}^{N} a_{i}(x, t)|\mathbf{m}(x, t)|^{\alpha_{i}}\right) \mathbf{m}(x, t)=-\nabla u(x, t) & (x, t) \in \Omega \times(0, T), \\
\phi(x) \partial_{t} u^{\lambda}(x, t)+\nabla \cdot \mathbf{m}(x, t)=f(x, t) & (x, t) \in \Omega \times(0, T), \\
u(x, t)=0 & (x, t) \in \partial \Omega \times(0, T), \\
u(x, 0)=u_{0}(x) & x \in \Omega .
\end{array}
$$

From now on the following assumptions will be needed

(H1) $0<\underline{\phi} \leq \phi(x) \leq \bar{\phi}<\infty ; f \in L^{\infty}\left(0, T ; L^{r^{*}}(\Omega)\right) ; u_{0} \in W_{0}^{1, s^{*}}(\Omega) \cap L^{r}(\Omega) ; a_{i}(\cdot, t) \in L^{\infty}(\Omega), i=0, \ldots, N$.

(H2) The coefficient functions and $\|f\|$ to be Lipschitz continuous in time, i.e., there exists a constant $L$ such that, for every $0 \leq t_{1} \leq t_{2} \leq T$,

$$
\left\|a_{i}\left(t_{1}\right)-a_{i}\left(t_{2}\right)\right\|_{L^{\infty}} \leq L\left|t_{1}-t_{2}\right|, \quad \text { and } \quad\left\|f\left(t_{1}\right)-f\left(t_{2}\right)\right\|_{L^{r^{*}}} \leq L\left|t_{1}-t_{2}\right| .
$$

(H3) The degree of Forchheimer polynomial $F$ satisfies $\alpha_{N}=\operatorname{deg}(F) \leq \gamma$. It equivalents to $r \leq s^{*}$. 
5.1. A priori estimates for the solutions of the semi-discrete problems. As mentioned above we use the technique of semi-discretization in time (see in [21]) to show the existence of solutions of the transient problem (5.1). The existence and uniqueness of the solutions to the semi-discrete problems has been established in Section 4 . In the next step, we consider the limit $h \rightarrow 0$. Similar to the regularized technique employed in the last two sections, we derive a priori estimates for the solutions of the semidiscrete problems, which are independent of $h$.

We investigate the semi-discrete problem (4.2) for homogeneous Dirichlet boundary condition. In this case problem (4.2) can read as the follow. Find $\left(\mathbf{m}^{j}, u^{j}\right) \in W(\operatorname{div}, \Omega) \times L^{r}(\Omega) \equiv V \times Q$, such that

$$
\begin{aligned}
a\left(\mathbf{m}^{j}, \mathbf{v}\right)-b\left(\mathbf{v}, u^{j}\right)=0 & \text { for all } \mathbf{v} \in V, \\
\left(\phi \frac{\left(u^{j}\right)^{\lambda}-\left(u^{j-1}\right)^{\lambda}}{h}, q\right)+b\left(\mathbf{m}^{j}, q\right)=\left(f^{j}, q\right) & \text { for all } q \in Q .
\end{aligned}
$$

Lemma 5.1. For sufficiently small $h<2^{-1} \underline{\phi} \lambda$, there exists $C>0$ independent of $h$ and $J$ such that

$$
\left\|u^{j}\right\|_{L^{r}}+\left\|\left(u^{j}\right)^{\lambda}\right\|_{L^{r^{*}}}+\left\|\mathbf{m}^{j}\right\|+\left\|\mathbf{m}^{j}\right\|_{L^{s}} \leq C \quad \text { for all } j=1,2, \ldots, J
$$

Proof. Choosing $(\mathbf{v}, q)=\left(\mathbf{m}^{j}, u^{j}\right)$ in 5.2 and adding the resulting equations yields

$$
a\left(\mathbf{m}^{j}, \mathbf{m}^{j}\right)+\left(\phi \frac{\left(u^{j}\right)^{\lambda}-\left(u^{j-1}\right)^{\lambda}}{h}, u^{j}\right)=\left(f^{j}, u^{j}\right) .
$$

From (2.9) we have

$$
\left(\phi \frac{\left(u^{j}\right)^{\lambda}-\left(u^{j-1}\right)^{\lambda}}{h}, u^{j}\right) \geq \frac{1}{h r^{*}}\left(\phi,\left(u^{j}\right)^{r}-\left(u^{j-1}\right)^{r}\right) .
$$

Due to the fact that $a\left(\mathbf{m}^{j}, \mathbf{m}^{j}\right) \geq 0$ and

$$
\left(f^{j}, u^{j}\right) \leq \frac{1}{r^{*}}\left\|f^{j}\right\|_{L^{r^{*}}}^{r^{*}}+\frac{1}{r}\left\|u^{j}\right\|_{L^{r}}^{r} \leq \frac{1}{r^{*}}\left\|f^{j}\right\|_{L^{r^{*}}}^{r^{*}}+\frac{1}{\underline{\phi} r}\left(\phi,\left(u^{j}\right)^{r}\right),
$$

it may be concluded that

$$
\left(\phi,\left(u^{j}\right)^{r}\right)-\left(\phi,\left(u^{j-1}\right)^{r}\right) \leq h\left\|f^{j}\right\|_{L^{r^{*}}}^{r^{*}}+\frac{h r^{*}}{\underline{\phi} r}\left(\phi,\left(u^{j}\right)^{r}\right) .
$$

If $h$ sufficient small so that $\ell h=\frac{h r^{*}}{\underline{\phi} r}=\frac{h}{\underline{\phi} \lambda}<1$, which gives $h<\underline{\phi} \lambda$, then

$$
\left(\phi,\left(u^{j}\right)^{r}\right) \leq \frac{1}{1-\ell h}\left(\left(\phi,\left(u^{j-1}\right)^{r}\right)+h\left\|f^{j}\right\|_{L^{r^{*}}}^{r^{*}}\right)
$$

By induction we find that

$$
\left(\phi,\left(u^{j}\right)^{r}\right) \leq(1-\ell h)^{-j}\left(\left(\phi,\left(u^{0}\right)^{r}\right)+\sum_{i=1}^{j}(1-\ell h)^{-i+1} h\left\|f^{i}\right\|_{L^{r^{*}}}^{r^{*}}\right) .
$$

Note that $(1-\ell h)^{-j} \leq e^{\frac{\ell T}{1-\ell h}}<e^{2 \ell T}$ for all $h<1 /(2 \ell)=\frac{1}{2} \underline{\phi} \lambda$, it follows from above inequality that

$$
\underline{\phi}\left\|u^{j}\right\|_{L^{r}}^{r} \leq\left(\phi,\left(u^{j}\right)^{r}\right) \leq e^{2 \ell T}\left(\bar{\phi}\left\|u^{0}\right\|_{L^{r}}^{r}+T\|f\|_{L^{\infty}\left(0, T ; L^{r^{*}}\right)}^{r^{*}}\right) .
$$


This leads to

$$
\left\|u^{j}\right\|_{L^{r}} \leq C_{1}
$$

where $C_{1}=\left(\underline{\phi}^{-1} e^{2 \ell T}\left(\bar{\phi}\left\|u^{0}\right\|_{L^{r}}^{r}+T\|f\|_{L^{\infty}\left(0, T ; L^{*}\right)}^{r^{*}}\right)\right)^{1 / r}$. It follows easily that

$$
\left\|\left(u^{j}\right)^{\lambda}\right\|_{L^{*^{*}}}=\left\|u^{j}\right\|_{L^{r}}^{\lambda} \leq C_{1}^{\lambda}
$$

Using the test function $q=u^{j}-u^{j-1}$, we obtain the from the second equation in 5.2 that

$$
\left(\phi \frac{\left(u^{j}\right)^{\lambda}-\left(u^{j-1}\right)^{\lambda}}{h}, u^{j}-u^{j-1}\right)+b\left(\mathbf{m}^{j}, u^{j}-u^{j-1}\right)=\left(f^{j}, u^{j}-u^{j-1}\right) .
$$

Now taking $\mathbf{v}=\mathbf{m}^{j}$ at time step $j$ and $j-1$ from the first equation in (5.2), we have

$$
a\left(\mathbf{m}^{j}, \mathbf{m}^{j}\right)-b\left(\mathbf{m}^{j}, u^{j}\right)=0, \quad \text { and } \quad a\left(\mathbf{m}^{j-1}, \mathbf{m}^{j}\right)-b\left(\mathbf{m}^{j}, u^{j-1}\right)=0,
$$

which implies that

$$
a\left(\mathbf{m}^{j}, \mathbf{m}^{j}\right)-a\left(\mathbf{m}^{j-1}, \mathbf{m}^{j}\right)=b\left(\mathbf{m}^{j}, u^{j}-u^{j-1}\right) .
$$

Combining (5.8) and (5.9) shows that

$$
\left(\phi \frac{\left(u^{j}\right)^{\lambda}-\left(u^{j-1}\right)^{\lambda}}{h}, u^{j}-u^{j-1}\right)+a\left(\mathbf{m}^{j}, \mathbf{m}^{j}\right)-a\left(\mathbf{m}^{j-1}, \mathbf{m}^{j}\right)=\left(f^{j}, u^{j}-u^{j-1}\right) .
$$

Summing up this equation for $k=1,2, \ldots, j$ yields

$$
\sum_{k=1}^{j}\left(\phi \frac{\left(u^{k}\right)^{\lambda}-\left(u^{k-1}\right)^{\lambda}}{h}, u^{k}-u^{k-1}\right)+a\left(\mathbf{m}^{k}, \mathbf{m}^{k}\right)-a\left(\mathbf{m}^{k-1}, \mathbf{m}^{k}\right)=\sum_{k=1}^{j}\left(f^{k}, u^{k}-u^{k-1}\right) .
$$

We will estimate (5.10) term by term.

The last term on the right hand side of (5.10) are bounded by using Hölder's inequality and (5.3)

$$
\begin{aligned}
\sum_{k=1}^{j}\left(f^{k}, u^{k}-u^{k-1}\right) & =\left(f^{j}, u^{j}\right)-\left(f^{1}, u^{0}\right)+\sum_{k=1}^{j-1}\left(f^{k}-f^{k+1}, u^{k}\right) \\
& \leq\left\|f^{j}\right\|_{L^{r^{*}}}\left\|u^{j}\right\|_{L^{r}}+\left\|f^{1}\right\|_{L^{r^{*}}}\left\|u^{0}\right\|_{L^{r}}+\sum_{k=1}^{j-1}\left\|f^{k}-f^{k+1}\right\|_{L^{r^{*}}}\left\|u^{k}\right\|_{L^{r}} \\
& \leq\|f\|_{L^{\infty}\left(0, T ; L^{r^{*}}\right)}\left(C_{1}+\left\|u^{0}\right\|_{L^{r}}\right)+C_{1} L T \leq\left(C_{1}+1\right)\left(\|f\|_{L^{\infty}\left(0, T ; L^{r^{*}}\right)}+\left\|u^{0}\right\|_{L^{r}}+L T\right)
\end{aligned}
$$

For the last two terms on the left hand side of (5.10), we rewrite as

$$
\sum_{k=1}^{j} a\left(\mathbf{m}^{k}, \mathbf{m}^{k}\right)-a\left(\mathbf{m}^{k-1}, \mathbf{m}^{k}\right)=\sum_{k=1}^{j} \sum_{i=0}^{N} \int_{\Omega}\left(a_{i}^{k}\left|\mathbf{m}^{k}\right|^{\alpha_{i}+2}-a_{i}^{k-1}\left|\mathbf{m}^{k-1}\right|^{\alpha_{i}} \mathbf{m}^{k-1} \cdot \mathbf{m}^{k}\right) d x .
$$

By Young's inequality

$$
\begin{aligned}
\sum_{i=0}^{N} a_{i}^{k-1}\left|\mathbf{m}^{k-1}\right|^{\alpha_{i}} \mathbf{m}^{k-1} \cdot \mathbf{m}^{k} & \leq \sum_{i=0}^{N} a_{i}^{k-1}\left(\frac{\alpha_{i}+1}{\alpha_{i}+2}\left|\mathbf{m}^{k-1}\right|^{\alpha_{i}+2}+\frac{1}{\alpha_{i}+2}\left|\mathbf{m}^{k}\right|^{\alpha_{i}+2}\right) \\
& =\sum_{i=0}^{N}\left(\frac{\left(\alpha_{i}+1\right) a_{i}^{k-1}}{\alpha_{i}+2}\left|\mathbf{m}^{j-1}\right|^{\alpha_{i}+2}+\frac{a_{i}^{k-1}-a_{i}^{k}}{\alpha_{i}+2}\left|\mathbf{m}^{k}\right|^{\alpha_{i}+2}+\frac{a_{i}^{k}}{\alpha_{i}+2}\left|\mathbf{m}^{k}\right|^{\alpha_{i}+2}\right) .
\end{aligned}
$$


Substituting (5.13) into (5.12) yields

$$
\begin{gathered}
\sum_{k=1}^{j} a\left(\mathbf{m}^{k}, \mathbf{m}^{k}\right)-a\left(\mathbf{m}^{k-1}, \mathbf{m}^{k}\right) \geq \sum_{k=1}^{j} \sum_{i=0}^{N} \int_{\Omega}\left(\frac{\alpha_{i}+1}{\alpha_{i}+2}\left(a_{i}^{k}\left|\mathbf{m}^{k}\right|^{\alpha_{i}+2}-a_{i}^{k-1}\left|\mathbf{m}^{k-1}\right|^{\alpha_{i}+2}\right)+\frac{a_{i}^{k}-a_{i}^{k-1}}{\alpha_{i}+2}\left|\mathbf{m}^{k}\right|^{\alpha_{i}+2}\right) d x \\
=\sum_{i=0}^{N} \int_{\Omega} \frac{\alpha_{i}+1}{\alpha_{i}+2}\left(a_{i}^{0}\left|\mathbf{m}^{0}\right|^{\alpha_{i}+2}+a_{i}^{j}\left|\mathbf{m}^{j}\right|^{\alpha_{i}+2}\right) d x+\sum_{i=0}^{N} \sum_{k=1}^{j} \int_{\Omega} \frac{a_{i}^{k}-a_{i}^{k-1}}{\alpha_{i}+2}\left|\mathbf{m}^{k}\right|^{\alpha_{i}+2} d x \\
\geq \int_{\Omega}\left(\frac{1}{2} a_{0}^{j}\left|\mathbf{m}^{j}\right|^{2}+\frac{s-1}{s} a_{N}^{j}\left|\mathbf{m}^{j}\right|^{s}\right) d x+\sum_{i=0}^{N} \sum_{k=1}^{j} \int_{\Omega} \frac{a_{i}^{k}-a_{i}^{k-1}}{\alpha_{i}+2}\left|\mathbf{m}^{k}\right|^{\alpha_{i}+2} d x .
\end{gathered}
$$

Due to (2.8), the first term

$$
\sum_{k=1}^{j}\left(\phi \frac{\left(u^{k}\right)^{\lambda}-\left(u^{k-1}\right)^{\lambda}}{h}, u^{k}-u^{k-1}\right) \geq 0 .
$$

Substituting (5.11), (5.14) and (5.15) into (5.10) yields

$$
\begin{gathered}
\frac{1}{2} a_{0}^{j}\left\|\mathbf{m}^{j}\right\|_{L^{2}}^{2}+\frac{s-1}{s} a_{N}^{j}\left\|\mathbf{m}^{j}\right\|_{L^{s}}^{s} \leq-\sum_{i=0}^{N} \sum_{k=1}^{j} \int_{\Omega} \frac{a_{i}^{k}-a_{i}^{k-1}}{\alpha_{i}+2}\left|\mathbf{m}^{k}\right|^{\alpha_{i}+2} d x+\left(C_{1}+1\right)\left(\|f\|_{L^{\infty}\left(0, T ; L^{*}\right)}+\left\|u^{0}\right\|_{L^{r}}+L T\right) \\
\leq \frac{L}{2 \underline{a}} \sum_{i=0}^{N} \sum_{k=1}^{j} \int_{\Omega} h a_{i}^{k}\left|\mathbf{m}^{k}\right|^{\alpha_{i}+2} d x+\left(C_{1}+1\right)\left(\|f\|_{L^{\infty}\left(0, T ; L^{*}\right)}+\left\|u^{0}\right\|_{L^{r}}+L T\right) \\
\leq \frac{L}{2 \underline{a}} \sum_{k=1}^{j} h a\left(\mathbf{m}^{k}, \mathbf{m}^{k}\right)+\left(C_{1}+1\right)\left(\|f\|_{L^{\infty}\left(0, T ; L^{*}\right)}+\left\|u^{0}\right\|_{L^{r}}+L T\right) .
\end{gathered}
$$

On the other hand by (5.4),

$$
\begin{aligned}
\sum_{k=1}^{j} h a\left(\mathbf{m}^{k}, \mathbf{m}^{k}\right)=-\sum_{k=1}^{j}\left(\phi\left(\left(u^{k}\right)^{\lambda}-\left(u^{k-1}\right)^{\lambda}\right), u^{k}\right)+h\left(f^{k}, u^{k}\right) \\
\quad \leq \frac{1}{r} \sum_{k=1}^{j}\left(\phi,\left(u^{k-1}\right)^{r}-\left(u^{k}\right)^{r}\right)+h\left\|f^{k}\right\|_{r^{*}}\left\|u^{k}\right\|_{L^{r}} \leq \frac{1}{r}\left(\phi,\left(u^{0}\right)^{r}-\left(u^{j}\right)^{r}\right)+C_{1} \sum_{k=1}^{j} h\left\|f^{k}\right\|_{r^{*}} \\
\quad \leq \frac{1}{r} \bar{\phi}\left(\left\|u^{0}\right\|_{L^{r}}^{r}+\left\|u^{j}\right\|_{L^{r}}^{r}\right)+C_{1} T\|f\|_{L^{\infty}\left(0, T ; L^{*}\right)} \leq\left(\frac{\bar{\phi}}{r}+1\right)\left(C_{1}^{r}+1\right)\left(\left\|u^{0}\right\|_{L^{r}}^{r}+T\|f\|_{L^{\infty}\left(0, T ; L^{*}\right)}+1\right) .
\end{aligned}
$$

Consequently,

$$
\begin{aligned}
\frac{1}{2} a_{0}^{j}\left\|\mathbf{m}^{j}\right\|_{L^{2}}^{2}+\frac{s-1}{s} a_{N}^{j}\left\|\mathbf{m}^{j}\right\|_{L^{s}}^{s} \leq & \frac{L}{2 \underline{a}}\left(\frac{\bar{\phi}}{r}+1\right)\left(C_{1}^{r}+1\right)\left(\left\|u^{0}\right\|_{L^{r}}^{r}+T\|f\|_{L^{\infty}\left(0, T ; L^{*}\right)}\right) \\
& +\left(C_{1}+1\right)\left(\|f\|_{L^{\infty}\left(0, T ; L^{*}\right)}+\left\|u^{0}\right\|_{L^{r}}+L T\right) .
\end{aligned}
$$

The assertion (5.3) directly follows from (5.6), (5.7) and (5.17).

Lemma 5.2. For sufficiently small $h$, there exists $\mathcal{C}>0$ independent of $h$ and $J$ such that

$$
\sum_{j=1}^{J} h \int_{\Omega}\left|\frac{u^{j}-u^{j-1}}{h}\right|^{r} d x \leq \mathcal{C} .
$$


Proof. We rewrite (5.10) as the form

$$
\sum_{j=1}^{J} \int_{\Omega} \phi \frac{\left(u^{j}\right)^{\lambda}-\left(u^{j-1}\right)^{\lambda}}{h}\left(u^{j}-u^{j-1}\right) d x=\sum_{j=1}^{J} a\left(\mathbf{m}^{j-1}, \mathbf{m}^{j}\right)-a\left(\mathbf{m}^{j}, \mathbf{m}^{j}\right)+\left(f^{j}, u^{j}-u^{j-1}\right) .
$$

From (5.14) we have

$$
\begin{gathered}
\sum_{j=1}^{J} a\left(\mathbf{m}^{j-1}, \mathbf{m}^{j}\right)-a\left(\mathbf{m}^{j}, \mathbf{m}^{j}\right) \leq \sum_{j=1}^{J} \sum_{i=0}^{N} \int_{\Omega}\left(\frac{\alpha_{i}+1}{\alpha_{i}+2}\left(a_{i}^{j-1}\left|\mathbf{m}^{j-1}\right|^{\alpha_{i}+2}-a_{i}^{j}\left|\mathbf{m}^{j}\right|^{\alpha_{i}+2}\right)+\frac{a_{i}^{j-1}-a_{i}^{j}}{\alpha_{i}+2}\left|\mathbf{m}^{j}\right|^{\alpha_{i}+2}\right) d x \\
\leq \sum_{i=0}^{N}\left(\bar{a}\left(\left\|\mathbf{m}^{0}\right\|_{L^{s}}^{\alpha_{i}+2}+\left\|\mathbf{m}^{J}\right\|_{L^{s}}^{\alpha_{i}+2}\right)+\sum_{j=1}^{J}\left\|a_{i}^{j-1}-a_{i}^{j}\right\|_{L^{\infty}}\left\|\mathbf{m}^{j}\right\|_{L^{s}}^{\alpha_{i}+2}\right) \\
\leq(\bar{a}+1)(N+1)\left(1+\left\|\mathbf{m}^{0}\right\|_{L^{s}}^{\alpha_{N}+2}+C^{\alpha_{N}+2}+L T\left(1+C^{\alpha_{N}+2}\right)\right) .
\end{gathered}
$$

It follows from (5.19), (5.11), (5.20) and (5.3) that

$$
\sum_{j=1}^{J} \int_{\Omega} \frac{\phi h}{\left|u^{j}\right|^{1-\lambda}+\left|u^{j-1}\right|^{1-\lambda}}\left|\frac{u^{j}-u^{j-1}}{h}\right|^{2} d x \leq \sum_{j=1}^{J} \int_{\Omega} \phi \frac{\left(u^{j}\right)^{\lambda}-\left(u^{j-1}\right)^{\lambda}}{h}\left(u^{j}-u^{j-1}\right) d x \leq C_{2},
$$

where

$$
C_{2} \stackrel{\text { def }}{=} C\left(C_{1}+1\right)\left(\|f\|_{L^{\infty}\left(0, T ; L^{*}\right)}+\left\|u^{0}\right\|_{L^{r}}+L T\right)+(\bar{a}+1)(N+1)\left(1+\left\|\mathbf{m}^{0}\right\|_{L^{s}}^{\alpha_{N}+2}+C^{\alpha_{N}+2}+L T\left(1+C^{\alpha_{N}+2}\right)\right) .
$$

On the other hand, by Hölder's inequality

$$
\begin{aligned}
& \sum_{j=1}^{J} h \int_{\Omega}\left|\frac{u^{j}-u^{j-1}}{h}\right|^{r} d x \\
& \quad \leq \sum_{j=1}^{J}\left(\int_{\Omega} h\left|\frac{\phi}{\left|u^{j}\right|^{1-\lambda}+\left|u^{j-1}\right|^{1-\lambda}}\right|^{-r /(2-r)} d x\right)^{1-r / 2}\left(\int_{\Omega} \frac{\phi h}{\left|u^{j}\right|^{1-\lambda}+\left|u^{j-1}\right|^{1-\lambda}}\left|\frac{u^{j}-u^{j-1}}{h}\right|^{2} d x\right)^{r / 2} \\
& \quad \leq\left(\sum_{j=1}^{J} \int_{\Omega} h\left|\frac{\phi}{\left|u^{j}\right|^{1-\lambda}+\left|u^{j-1}\right|^{1-\lambda}}\right|^{-r /(2-r)} d x\right)^{1-r / 2}\left(\sum_{j=1}^{J} \int_{\Omega} \frac{\phi h}{\left|u^{j}\right|^{1-\lambda}+\left|u^{j-1}\right|^{1-\lambda}}\left|\frac{u^{j}-u^{j-1}}{h}\right|^{2} d x\right)^{r / 2} .
\end{aligned}
$$

Since

$$
\begin{aligned}
\sum_{j=1}^{J} \int_{\Omega} h\left|\frac{\phi}{\left|u^{j}\right|^{1-\lambda}+\left|u^{j-1}\right|^{1-\lambda}}\right|^{-r /(2-r)} d x & \leq \sum_{j=1}^{J} h \int_{\Omega} \phi^{-r /(2-r)}\left(\left|u^{j}\right|^{1-\lambda}+\left|u^{j-1}\right|^{1-\lambda}\right)^{r /(2-r)} d x \\
& \leq \sum_{j=1}^{J} h \underline{\phi}^{-r /(2-r)} 2^{2(r-1) /(2-r)}\left(\left\|u^{j}\right\|_{L^{r}}^{r}+\left\|u^{j-1}\right\|_{L^{r}}^{r}\right),
\end{aligned}
$$

we use (5.21) and (5.3) to conclude that

$$
\sum_{j=1}^{J} h \int_{\Omega}\left|\frac{u^{i}-u^{i-1}}{h}\right|^{r} d x \leq\left(2 C_{2}\right)^{r / 2} C^{r(1-r / 2)} T^{1-r / 2} \underline{\phi}^{-r / 2} .
$$


This completes the proof. $\square$

Next, we show that the mixed formulation (5.2) is equivalent to a variational formulation of the timediscretized parabolic equation. To this end, we recall the nonlinear mapping $K$ of (1.14). For fixed time $t=t_{j}$, we define the nonlinear mapping $K^{j}: \Omega \times \mathbb{R}^{+} \rightarrow \mathbb{R}^{+}$(see in (1.15) ) and its inverse defined by

$$
F^{j}(x, z)=a_{0}\left(x, t_{j}\right) z^{\alpha_{0}}+a_{1}\left(x, t_{j}\right) z^{\alpha_{1}}+\cdots+a_{N}\left(x, t_{j}\right) z^{\alpha_{N}}, \quad z \geq 0 .
$$

LeMma 5.3.

(i) If $u^{j} \in R(\Omega)=\left\{r \in L^{r}(\Omega), r=0\right.$ on $\left.\partial \Omega, \nabla r \in\left(L^{s^{*}}(\Omega)\right)^{d}\right\}$ is a solution of the variational formulation. Find $u^{j} \in R(\Omega)$ such that

$$
\left(\phi \frac{\left(u^{j}\right)^{\lambda}-\left(u^{j-1}\right)^{\lambda}}{h}, q\right)+\left(K^{j}\left(x,\left|\nabla u^{j}\right|\right) \nabla u^{j}, \nabla q\right)=\left(f^{j}, q\right) \quad \text { for all } q \in R(\Omega)
$$

then $\left(-K^{j}\left(x,\left|\nabla u^{j}\right|\right) \nabla u^{j}, u^{j}\right)$ is a solution of the mixed formulation (5.2).

(ii) If $\left(\mathbf{m}^{j}, u^{j}\right) \in W(\operatorname{div}, \Omega) \times \mathrm{L}^{\mathrm{r}}(\Omega)$ is a solution of the mixed formulation (5.2) then $u^{j}$ is a solution of the variational formulation (5.23). In particular, $u^{j} \in R(\Omega)$.

Proof.

(i) Let $u^{j}$ be a solution of (5.23). We define $\mathbf{m}^{j}=-K^{j}\left(x,\left|\nabla u^{j}\right|\right) \nabla u^{j}$. Then Green's formula yields

$$
\left(F^{j}\left(x,\left|\mathbf{m}^{j}\right|\right) \mathbf{m}^{j}, \mathbf{v}\right)=-\left(\nabla u^{j}, \mathbf{v}\right)=\left(u^{j}, \nabla \cdot \mathbf{v}\right) \quad \text { for all } \mathbf{v} \in V .
$$

This is the first equation in (5.2). To derive the second equation in (5.2), we consider (5.23) for $q \in \mathcal{D}(\Omega) \subset$ $R(\Omega)$

$$
\left(\phi \frac{\left(u^{j}\right)^{\lambda}-\left(u^{j-1}\right)^{\lambda}}{h}, q\right)-\left(\mathbf{m}^{j}, \nabla q\right)=\left(f^{j}, q\right),
$$

and then apply Green's formula we obtain

$$
\left(\phi \frac{\left(u^{j}\right)^{\lambda}-\left(u^{j-1}\right)^{\lambda}}{h}, q\right)+\left(\nabla \cdot \mathbf{m}^{j}, q\right)=\left(f^{j}, q\right) .
$$

Because $\mathcal{D}(\Omega)$ is densely embedded into $L^{r}(\Omega)$, the second equation in $(5.2)$ follows.

(ii) Let $\left(\mathbf{m}^{j}, u^{j}\right)$ be the solution of (5.2). Applying Green's formula implies

$$
\left(F^{j}\left(x,\left|\mathbf{m}^{j}\right|\right) \mathbf{m}^{j}, \mathbf{v}\right)=\left(\nabla \cdot \mathbf{v}, u^{j}\right)=\left(-\nabla u^{j}, \mathbf{v}\right) \quad \text { for all } \mathbf{v} \in(\mathcal{D}(\Omega))^{d} .
$$

Thus in the sense of distributions it holds $\nabla u^{j}=-F^{j}\left(x,\left|\mathbf{m}^{j}\right|\right) \mathbf{m}^{j} \in\left(L^{s *}(\Omega)\right)^{d}$.

Consequently, $u^{j} \in\left\{r \in L^{r}(\Omega), \nabla r \in\left(L^{s^{*}}(\Omega)\right)^{d}\right\}$ and $\mathbf{m}^{j}=-K^{j}\left(x,\left|\nabla u^{j}\right|\right) \nabla u^{j}$.

To prove that $u^{j}$ fulfills (5.23), we consider $q \in R(\Omega) \subset L^{r}(\Omega)$ in the first equation of (5.2). Using integration by parts, we have

$$
\left(f^{j}, q\right)=\left(\phi \frac{\left(u^{j}\right)^{\lambda}-\left(u^{j-1}\right)^{\lambda}}{h}, q\right)+\left(\nabla \cdot \mathbf{m}^{j}, q\right)=\left(\phi \frac{\left(u^{j}\right)^{\lambda}-\left(u^{j-1}\right)^{\lambda}}{h}, q\right)+\left(K^{j}\left(x,\left|\nabla u^{j}\right|\right) \nabla u^{j}, \nabla q\right) .
$$

Finally, we consider again the first equation of $(\overline{5.2})$ for $\mathbf{v} \in(\mathcal{D}(\bar{\Omega}))^{d}$. Using integration by parts, we obtain

$$
0=-\left(F^{j}\left(x,\left|\mathbf{m}^{j}\right|\right) \mathbf{m}^{j}, \mathbf{v}\right)+\left(\nabla \cdot \mathbf{v}, u^{j}\right)=\left(\nabla u^{j}, \mathbf{v}\right)+\left(\nabla \cdot \mathbf{v}, u^{j}\right)=\int_{\partial \Omega} u^{j}(\mathbf{v} \cdot v) d \sigma
$$


Consequently, $u^{j}=0$ on $\partial \Omega$, i.e., $u^{j} \in R(\Omega)$.

Using this equivalence, we obtain a bound for $u^{j}$ in the norm of $R(\Omega)$ defined by

$$
\|r\|_{R}=\|r\|_{L^{r}}+\|\nabla r\|_{L^{*}}
$$

Lemma 5.4. For sufficiently small $h$, there is a constants $C>0$ independent of $h$ and $J$, such that

$$
\left\|u^{j}\right\|_{R}+\left\|\frac{\left(u^{j}\right)^{\lambda}-\left(u^{j-1}\right)^{\lambda}}{h}\right\|_{R^{\prime}}+\left\|\nabla \cdot \mathbf{m}^{j}\right\|_{R^{\prime}} \leq C \quad \text { for all } j=0,1,2, \ldots, J .
$$

Proof. To verify $\left\|u^{j}\right\|_{R}$ is bounded, it is sufficient to use (5.3) together with the observation that

$$
\begin{aligned}
\left\|\nabla u^{j}\right\|_{L^{s^{*}}}^{s^{*}}=\left\|F^{j}\left(x,\left|\mathbf{m}^{j}\right|\right) \mathbf{m}^{j}\right\|_{L^{s^{*}}}^{s^{*}} & \leq C \sum_{i=0}^{N} \int_{\Omega}\left|\mathbf{m}^{j}\right|^{\left(\alpha_{i}+1\right) s^{*}} d x \\
& \leq C \sum_{i=0}^{N}\left\|\mathbf{m}^{j}\right\|_{L^{s}}^{\left(\alpha_{i}+1\right) s^{*}} \leq C N\left(\left\|\mathbf{m}^{j}\right\|_{L^{s}}^{s^{*}}+\left\|\mathbf{m}^{j}\right\|_{L^{s}}^{\left(\alpha_{N}+1\right) s^{*}}\right) .
\end{aligned}
$$

We next to prove that $\left\|\frac{\left(u^{j}\right)^{\lambda}-\left(u^{j-1}\right)^{\lambda}}{h}\right\|_{R^{\prime}}$ is bounded.

By means of (5.23), we have for all $q \in R(\Omega)$,

$$
\begin{aligned}
\left|\left(\phi \frac{\left(u^{j}\right)^{\lambda}-\left(u^{j-1}\right)^{\lambda}}{h}, q\right)\right|=\left|\left(f^{j}, q\right)-\left(K^{j}\left(x,\left|\nabla u^{j}\right|\right) \nabla u^{j}, q\right)\right| & =\left|\left(f^{j}, q\right)+\left(\mathbf{m}^{j}, \nabla q\right)\right| \\
& \leq\left(\left\|f^{j}\right\|_{L^{r^{*}}}+\left\|\mathbf{m}^{j}\right\|_{L^{s}}\right)\|q\|_{R} .
\end{aligned}
$$

Thanks to the boundedness of the function $\phi$ and $(5.3)$ we see that

$$
\left\|\frac{\left(u^{j}\right)^{\lambda}-\left(u^{j-1}\right)^{\lambda}}{h}\right\|_{R^{\prime}} \leq C\left(\left\|f^{j}\right\|_{L^{r^{*}}}+\left\|\mathbf{m}^{j}\right\|_{L^{s}}\right) \leq C
$$

which is part of conclusion (5.24).

From the second equation of (5.2) yields

$$
\left|\left(\nabla \cdot \mathbf{m}^{j}, q\right)\right|=\left|\left(f^{j}, q\right)-\left(\phi \frac{\left(u^{j}\right)^{\lambda}-\left(u^{j-1}\right)^{\lambda}}{h}, q\right)\right| \leq\left(\left\|f^{j}\right\|_{L^{*^{*}}}+\bar{\phi}\left\|\frac{\left(u^{j}\right)^{\lambda}-\left(u^{j-1}\right)^{\lambda}}{h}\right\|_{L^{*}}\right)\|q\|_{R},
$$

which implies that

$$
\left\|\nabla \cdot \mathbf{m}^{j}\right\|_{R^{\prime}} \leq\left\|f^{j}\right\|_{L^{*}}+\bar{\phi}\left\|\frac{\left(u^{j}\right)^{\lambda}-\left(u^{j-1}\right)^{\lambda}}{h}\right\|_{L^{m^{*}}} \leq\left\|f^{j}\right\|_{L^{m^{*}}}+\bar{\phi}\left\|\frac{\left(u^{j}\right)^{\lambda}-\left(u^{j-1}\right)^{\lambda}}{h}\right\|_{R^{\prime}} \leq C .
$$

The proof is complete.

5.2. Solvability of the continuous problem. Due to the existence of unique solutions to the semidiscrete mixed formulation (5.2), we obtain for every $J \in \mathbb{N}$ a $J+1$-tuple of solutions $\left(\mathbf{m}^{j}, u^{j}\right)_{j=0, \ldots, J} \in$ $\left(W(\operatorname{div}, \Omega) \times \mathrm{L}^{\mathrm{r}}(\Omega)\right)^{\mathrm{J}+1}$. We denote these $J+1$-tuples with $\mathbf{m}_{h}:=\left(\mathbf{m}^{j}\right)_{j=0, \ldots, J} \in(W(\operatorname{div}, \Omega))^{\mathrm{J}+1}$ and $u_{h}:=$ $\left(u^{j}\right)_{j=0, \ldots, J} \in\left(L^{r}(\Omega)\right)^{J+1}$. We define step function by

$$
\pi u_{h}(t)=\sum_{j=1}^{J} \chi_{j}(t) u^{j} \in L^{\infty}(0, T ; R(\Omega)),
$$


where $\chi_{j}(t)$ is the characteristic function on the interval $\left(t_{j-1}, t_{j}\right.$ ] for $j=1,2, \ldots J$. We also defined a piecewise linear (in time) functions

$$
\Pi u_{h}(t)=\sum_{j=1}^{J} \chi_{j}(t)\left(\frac{u^{j}-u^{j-1}}{h}\left(t-t_{j}\right)+u^{j}\right) .
$$

In addition, we use piecewise constant approximations $a_{i h}$ and $f_{h}$ of the coefficient functions $a_{i}$ and $f$, and piecewise constant operators $F_{h}$ and $K_{h}$. According to Lemmas 5.1 and 5.4 the following bounds hold for sufficiently small $h$.

$$
\begin{array}{ll}
\left\|\pi u_{h}\right\|_{L^{\infty}(0, T ; R(\Omega))} \leq C, & \left\|\partial_{t} \Pi u_{h}\right\|_{L^{r}\left(0, T ; L^{r}(\Omega)\right)} \leq C, \\
\left\|\left(\pi u_{h}\right)^{\lambda}\right\|_{L^{\infty}\left(0, T ; L^{*}(\Omega)\right)} \leq C, & \left\|\partial_{t} \Pi u_{h}^{\lambda}\right\|_{L^{\infty}\left(0, T ; R^{\prime}(\Omega)\right)} \leq C, \\
\left\|\pi \mathbf{m}_{h}\right\|_{L^{\infty}\left(0, T ;\left(L^{s}(\Omega)\right)^{d}\right)} \leq C, & \left\|\pi \nabla \cdot \mathbf{m}_{h}\right\|_{L^{\infty}\left(0, T ; R^{\prime}(\Omega)\right)} \leq C, \\
\left\|F\left(x,\left|\pi \mathbf{m}_{h}\right|\right) \pi \mathbf{m}_{h}\right\|_{L^{\infty}\left(0, T ;\left(L^{*}(\Omega)\right)^{d}\right)} \leq C, & \left\|u^{J}\right\|_{L^{r}(\Omega)} \leq C .
\end{array}
$$

Thus the exist a subsequences, again indexed by $h$, that converge in corresponding weak*-topology; in detail

$$
\begin{array}{ll}
\pi u_{h} \stackrel{*}{\rightarrow} u \text { in } L^{\infty}(0, T ; R(\Omega)), & \partial_{t} \Pi u_{h} \stackrel{*}{\rightarrow} u^{\prime} \text { in } L^{\infty}\left(0, T ; L^{r}(\Omega)\right) \\
\left(\pi u_{h}\right)^{\lambda} \stackrel{*}{\rightarrow} U \text { in } L^{\infty}\left(0, T ; L^{r^{*}}(\Omega)\right), & \partial_{t} \Pi u_{h}^{\lambda} \stackrel{*}{\rightarrow} U^{\prime} \text { in } L^{\infty}\left(0, T ; R^{\prime}(\Omega)\right) \\
\pi \mathbf{m}_{h} \stackrel{*}{\rightarrow} \mathbf{m} \text { in } L^{\infty}\left(0, T ;\left(L^{r}(\Omega)\right)^{d}\right), & \pi \nabla \cdot \mathbf{m}_{h} \stackrel{*}{\rightarrow} \overline{\mathbf{m}} \text { in } L^{\infty}\left(0, T ; R^{\prime}(\Omega)\right) \\
F\left(x,\left|\pi \mathbf{m}_{h}\right|\right) \pi \mathbf{m}_{h} \stackrel{*}{\rightarrow} \hat{F} \text { in } L^{\infty}\left(0, T ;\left(L^{s^{*}}(\Omega)\right)^{d}\right), & u^{J} \rightarrow u_{T} \text { in } L^{r}(\Omega) .
\end{array}
$$

Lemma 5.5. (i) The identity $U=u^{\lambda}$ hold in the sense of distribution from $(0, T)$ to $L^{r^{*}}(\Omega)$.

(ii) The identity $u^{\prime}=\partial_{t} u$ holds in the sense of distribution from $(0, T)$ to $L^{r}(\Omega)$. That is for all $\varphi \in$ $\mathcal{D}((0, T))$,

$$
\int_{0}^{T} u^{\prime}(t) \varphi(t) d t=-\int_{0}^{T} u(t) \varphi^{\prime}(t) d t \text { in } L^{r}(\Omega) .
$$

(iii) The identity $U^{\prime}=\partial_{t} U$ holds in the sense of distribution from $(0, T)$ to $R^{\prime}$. That is for all $\varphi \in$ $\mathcal{D}((0, T))$,

$$
\int_{0}^{T} U^{\prime}(t) \varphi(t) d t=-\int_{0}^{T} U(t) \varphi^{\prime}(t) d t \text { in } R^{\prime}(\Omega)
$$

(iv) The identity $\overline{\mathbf{m}}=\nabla \cdot \mathbf{m}$ hold in the sense of distribution on $\Omega$ hold for almost everywhere in $(0, T)$. That is for all $\psi \in \mathcal{D}(\Omega)$,

$$
(\overline{\mathbf{m}}, \psi)=-(\mathbf{m}, \nabla \psi) \text { a.e in }(0, T) .
$$

(v) The identity $\hat{F}=-\nabla u$ hold in $L^{\infty}\left(0, T ;\left(L^{S^{*}}(\Omega)\right)^{d}\right)$. That is for all $\mathbf{v} \in L^{1}\left(0, T ;\left(L^{s}(\Omega)\right)^{d}\right)$,

$$
\int_{0}^{T}(\hat{F}, \mathbf{v}) d t=-\int_{0}^{T}(\nabla u, \mathbf{v}) d t
$$


Proof. (i) Since $\pi u_{h} \in L^{\infty}(0, T ; R(\Omega))$, $\Pi u_{h} \in L^{\infty}(0, T ; R(\Omega))$. In particular, $\Pi u_{h} \in L^{r}\left(0, T ; L^{r}(\Omega)\right)$ and $\partial_{x_{i}}\left(\Pi u_{h}\right) \in L^{r}\left(0, T ; L^{r}(\Omega)\right)$. Due to $(5.18), \partial_{t} \Pi u_{h} \in L^{r}\left(0, T ; L^{r}(\Omega)\right)$. This implies $\Pi u_{h} \in W^{1, r}((0, T) \times \Omega)$. The Rellich- Kondrachov theorem yields that $W^{1, r}((0, T) \times \Omega)$ is compactly embedded in $L^{r}((0, T) \times \Omega)$. There is the subsequence $\Pi u_{h} \rightarrow u$ strongly in $L^{r}((0, T) \times \Omega)$. Thus $\lim _{h \rightarrow 0} \Pi u_{h}^{\lambda}=u^{\lambda}$ a.e in $L^{r^{*}}((0, T) \times \Omega)$. Since $\Pi u_{h}^{\lambda}$ is bounded in $L^{r^{*}}((0, T) \times \Omega)$ we conclude that $\Pi u_{h}^{\lambda}$ converge weakly to $u^{\lambda}$ in $L^{r^{*}}((0, T) \times \Omega)$ that is for all $\varphi \in L^{r}((0, T), \Omega)$,

$$
\lim _{h \rightarrow 0} \int_{0}^{T}\left(\Pi u_{h}^{\lambda}, \varphi\right) d t=\int_{0}^{T}\left(u^{\lambda}, \varphi\right) d t
$$

On the other hand,

$$
\lim _{h \rightarrow 0} \int_{0}^{T}\left(\Pi u_{h}^{\lambda}, \varphi\right) d t=\lim _{h \rightarrow 0} \int_{0}^{T}\left(\pi u_{h}^{\lambda}, \varphi\right) d t=\lim _{h \rightarrow 0} \int_{0}^{T}\left(\left(\pi u_{h}\right)^{\lambda}, \varphi\right) d t=\int_{0}^{T}(U, \varphi) d t .
$$

Then the assertion follows.

(ii) Let $\varphi \in \mathcal{D}(0, T)$ then

$$
\int_{0}^{T} u^{\prime}(t) \varphi(t) d t=\lim _{h \rightarrow 0} \int_{0}^{T} \partial_{t} \Pi u_{h} \varphi(t) d t=-\lim _{h \rightarrow 0} \int_{0}^{T} \Pi u_{h} \varphi^{\prime}(t) d t=-\lim _{h \rightarrow 0} \int_{0}^{T} \pi u_{h} \varphi^{\prime}(t) d t=-\int_{0}^{T} u \varphi^{\prime}(t) d t
$$

(iii) Similar to (ii)

(iv) Let $\psi \in \mathcal{D}(\Omega)$ and $\varphi \in \mathcal{D}(0, T)$ then

$$
\int_{0}^{T}(\overline{\mathbf{m}}, \psi) \varphi(t) d t=\lim _{h \rightarrow 0} \int_{0}^{T}\left(\pi \nabla \cdot \mathbf{m}_{h}, \psi\right) \varphi(t) d t=-\lim _{h \rightarrow 0} \int_{0}^{T}\left(\pi \mathbf{m}_{h}, \nabla \psi\right) \varphi(t) d t=-\int_{0}^{T}(\mathbf{m}, \nabla \psi) \varphi(t) d t .
$$

(v) For all $\mathbf{v} \in L^{1}\left(0, T,\left(L^{s}(\Omega)\right)^{d}\right)$,

$$
\int_{0}^{T}(\hat{F}, \mathbf{v}) d t=\lim _{h \rightarrow 0} \int_{0}^{T}\left(F\left(x,\left|\pi \mathbf{m}_{h}\right|\right) \pi \mathbf{m}_{h}, \mathbf{v}\right) d t=-\lim _{h \rightarrow 0} \int_{0}^{T}\left(\pi \nabla u_{h}, \mathbf{v}\right) d t=-\int_{0}^{T}(\nabla u, \mathbf{v}) d t .
$$

The proof is complete.

Lemma 5.6. The following identity holds in $L^{\infty}\left(0, T ; L^{r}(\Omega)\right)$

$$
\phi \partial_{t} u^{\lambda}+\nabla \cdot \mathbf{m}=f
$$

Furthermore $u(x, 0)=u_{0}(x)$, and $u(x, T)=u_{T}$.

Proof. For $\varphi \in \mathcal{D}([0, T])$ we defined the step function $\varphi_{h}$ by

$$
\varphi_{h}(t)=\left\{\begin{array}{ll}
\varphi\left(t_{j-1}\right) & \text { if } t_{j-1} \leq t<t_{j}, j=1, \ldots, J \\
\varphi(T) & \text { if } t=t_{J}
\end{array} .\right.
$$

Using the test function $q=\psi \in \mathcal{D}(\Omega)$ in the second equation of (5.2), multiplying by $h \varphi\left(t_{j-1}\right)$ and summing up on $j=1, \ldots, J$, we obtain

$$
\sum_{j=1}^{J}\left(\phi \frac{\left(u^{j}\right)^{\lambda}-\left(u^{j-1}\right)^{\lambda}}{h}, \psi\right) h \varphi\left(t_{j-1}\right)+\left(\nabla \cdot \mathbf{m}^{j}, \psi\right) h \varphi\left(t_{j-1}\right)=\sum_{j=1}^{J}\left(f^{j}, \psi\right) h \varphi\left(t_{j-1}\right) .
$$


Using the piecewise constant function $\pi$ this reads

$$
\int_{0}^{T}\left(\phi \partial_{t} \Pi u_{h}^{\lambda}, \psi\right) \varphi_{h} d t+\int_{0}^{T}\left(\pi \nabla \cdot \mathbf{m}_{h}, \psi\right) \varphi_{h} d t=\int_{0}^{T}(\pi f, \psi) \varphi_{h} d t .
$$

Since $\psi \varphi_{h}$ converges strongly to $\psi \varphi$ in $L^{1}(0, T ; R(\Omega))$. Hence a passage to the limit $h \rightarrow 0$ implies that

$$
\int_{0}^{T}\left(\phi \partial_{t} u^{\lambda}, \psi\right) \varphi d t+\int_{0}^{T}(\nabla \cdot \mathbf{m}, \psi) \varphi d t=\int_{0}^{T}(f, \psi) \varphi d t
$$

The set $\{\psi \varphi, \psi \in \mathcal{D}(\Omega), \varphi \in \mathcal{D}(\overline{(0, T)})\}$ is dense subset of $L^{1}(0, T ; R(\Omega))$. Thus the identity (5.32) is established.

To prove the remaining two identities we rewrite $(5.33)$ as form

$$
\begin{aligned}
-\sum_{j=1}^{J} h\left(\phi\left(u^{j}\right)^{\lambda}, \psi\right) \frac{\varphi\left(t_{j}\right)-\varphi\left(t_{j-1}\right)}{h}+\sum_{j=1}^{J} h & \left(\nabla \cdot \mathbf{m}^{j}, \psi\right) \varphi\left(t_{j-1}\right) \\
& =\sum_{j=1}^{J} h\left(f^{j}, \psi\right) \varphi\left(t_{j-1}\right)-\left(\phi\left(u^{J}\right)^{\lambda}, \psi\right) \varphi(T)+\left(\phi\left(u^{0}\right)^{\lambda}, \psi\right) \varphi(0),
\end{aligned}
$$

which is

$$
-\int_{0}^{T}\left(\phi \pi u_{h}^{\lambda}, \psi\right) \partial_{t} \Pi \varphi d t+\int_{0}^{T}\left(\pi \nabla \cdot \mathbf{m}_{h}, \psi\right) \varphi_{h} d t=\int_{0}^{T}(\pi f, \psi) \varphi_{h} d t-\left(\phi\left(u^{J}\right)^{\lambda}, \psi\right) \varphi(T)+\left(\phi\left(u^{0}\right)^{\lambda}, \psi\right) \varphi(0) .
$$

Passing to the limit $h \rightarrow 0$ we obtain

$$
-\int_{0}^{T}\left(\phi u^{\lambda}, \psi\right) \partial_{t} \varphi d t+\int_{0}^{T}(\nabla \cdot \mathbf{m}, \psi) \varphi d t=\int_{0}^{T}(f, \psi) \varphi d t-\left(\phi\left(u_{T}\right)^{\lambda}, \psi\right) \varphi(T)+\left(\phi\left(u^{0}\right)^{\lambda}, \psi\right) \varphi(0) .
$$

On the other hand, partial integration of (5.34) yields

$$
-\int_{0}^{T}\left(\phi u^{\lambda}, \psi\right) \partial_{t} \varphi d t+\int_{0}^{T}(\nabla \cdot \mathbf{m}, \psi) \varphi d t=\int_{0}^{T}(f, \psi) \varphi d t-\left(\phi u^{\lambda}(T), \psi\right) \varphi(T)+\left(\phi u^{\lambda}(0), \psi\right) \varphi(0) .
$$

We compare (5.35) and (5.36) to obtain

$$
\left(\phi\left(u^{\lambda}(0)-\left(u^{0}\right)^{\lambda}\right), \psi\right) \varphi(0)=\left(\phi\left(u^{\lambda}(T)-u_{T}^{\lambda}\right), \psi\right) \varphi(T) .
$$

Since $\varphi(0)$ and $\varphi(T)$ are arbitrary, we have

$$
\left(\phi\left(u^{\lambda}(0)-\left(u^{0}\right)^{\lambda}\right), \psi\right)=0=\left(\phi\left(u^{\lambda}(T)-u_{T}^{\lambda}\right), \psi\right) .
$$

Thus $u(0)=u^{0}$ and $u(T)=u_{T}$.

Lemma 5.7. The limit $\mathbf{m}$ of $\pi \mathbf{m}_{h}$ and $\hat{F}$ of $F\left(x, t, \pi \mathbf{m}_{h}\right)$ satisfy $\hat{F}=F(x, t,|\mathbf{m}|) \mathbf{m}$ in $L^{\infty}\left(0, T ;\left(L^{s^{*}}(\Omega)\right)^{d}\right)$. That means

$$
\int_{0}^{T}(\hat{F}, \mathbf{v}) d t=\int_{0}^{T}(F(x, t,|\mathbf{m}|) \mathbf{m}, \mathbf{v}) d t \quad \text { for all } \mathbf{v} \in L^{1}\left(0, T ;\left(L^{s}(\Omega)\right)^{d}\right) .
$$


To show this, we need an auxiliary result, a particular result of Lemma 1.2 in [21].

Proposition 5.8. The limit $u$ of $\pi u_{h}$ satisfy

$$
\int_{0}^{T}\left(\phi \partial_{t} u^{\lambda}(t), u(t)\right) d t=\frac{1}{r^{*}} \int_{\Omega} \phi u^{r}(T)-\phi u^{r}(0) d x
$$

Proof. Equation (5.4) rewrite as

$$
\left(F\left(x, t,\left|\mathbf{m}^{j}\right|\right) \mathbf{m}^{j}, \mathbf{m}^{j}\right)+\left(\phi \frac{\left(u^{j}\right)^{\lambda}-\left(u^{j-1}\right)^{\lambda}}{h}, u^{j}\right)=\left(f^{j}, u^{j}\right) .
$$

Since $\left(\phi \frac{\left(u^{j}\right)^{\lambda}-\left(u^{j-1}\right)^{\lambda}}{h}, u^{j}\right) \geq \frac{1}{h r^{*}}\left(\phi,\left(u^{j}\right)^{r}-\left(u^{j-1}\right)^{r}\right)$,

$$
\left(F\left(x, t,\left|\mathbf{m}^{j}\right|\right) \mathbf{m}^{j}, \mathbf{m}^{j}\right)+\frac{1}{h r^{*}}\left(\phi,\left(u^{j}\right)^{r}-\left(u^{j-1}\right)^{r}\right) \leq\left(f^{j}, u^{j}\right) .
$$

Multiplying $h$ and summing up $j=1, \ldots, J$, we obtain

$$
\int_{0}^{T}\left(F\left(x, t,\left|\pi \mathbf{m}_{h}\right|\right) \pi \mathbf{m}_{h}, \pi \mathbf{m}_{h}\right) d t+\frac{1}{r^{*}}\left(\phi,\left(u^{J}\right)^{r}-\left(u^{0}\right)^{r}\right) \leq \int_{0}^{T}\left(\pi f_{h}, \pi u_{h}\right) d t .
$$

We take the limit inferior to conclude that

$$
\liminf _{h \rightarrow 0} \int_{0}^{T}\left(F\left(x, t,\left|\pi \mathbf{m}_{h}\right|\right) \pi \mathbf{m}_{h}, \pi \mathbf{m}_{h}\right) d t+\frac{1}{r^{*}}\left(\phi,\left(u^{J}\right)^{r}-\left(u^{0}\right)^{r}\right) \leq \int_{0}^{T}(f, u) d t .
$$

Using the result of Proposition 5.8, we find that

$$
\liminf _{h \rightarrow 0} \int_{0}^{T}\left(F\left(x, t,\left|\pi \mathbf{m}_{h}\right|\right) \pi \mathbf{m}_{h}, \pi \mathbf{m}_{h}\right) d t+\int_{0}^{T}\left(\phi \partial_{t} u^{\lambda}, u\right) d t \leq \int_{0}^{T}(f, u) d t .
$$

On the other hand, Lemma 5.6 gives

$$
\int_{0}^{T}\left(\phi \partial_{t} u^{\lambda}, u\right) d t+\int_{0}^{T}(\nabla \cdot \mathbf{m}, u) d t=\int_{0}^{T}(f, u) d t .
$$

From (5.30) and (5.31) in Lemma5.5 we have

$$
\int_{0}^{T}(\nabla \cdot \mathbf{m}, u) d t=-\int_{0}^{T}(\mathbf{m}, \nabla u) d t=\int_{0}^{T}(\mathbf{m}, \hat{F}) d t .
$$

Therefore,

$$
\liminf _{h \rightarrow 0} \int_{0}^{T}\left(F\left(x, t,\left|\pi \mathbf{m}_{h}\right|\right) \pi \mathbf{m}_{h}, \pi \mathbf{m}_{h}\right) d t \leq \int_{0}^{T}(\mathbf{m}, \hat{F}) d t .
$$

We have shown that for arbitrary $\mathbf{v} \in L^{\infty}\left(0, T ;\left(L^{s}(\Omega)\right)^{d}\right)$

$$
\int_{0}^{T}(\hat{F}-F(x, t,|\mathbf{v}| \mathbf{v}), \mathbf{m}-\mathbf{v}) d t \geq \liminf _{h \rightarrow 0} \int_{0}^{T}\left(F\left(x, t,\left|\pi \mathbf{m}_{h}\right|\right) \pi \mathbf{m}_{h}-F(x, t,|\mathbf{v}|) \mathbf{v}, \pi \mathbf{m}_{h}-\mathbf{v}\right) d t \geq 0 .
$$

Choose $\mathbf{v}=\mathbf{m}-\lambda \varphi, \lambda>0, \varphi \in L^{\infty}\left(0, T ;\left(L^{s}(\Omega)\right)^{d}\right)$

$$
\int_{0}^{T}(\hat{F}-F(x, t,|\mathbf{m}-\lambda \varphi|)(\mathbf{m}-\lambda \varphi), \lambda \varphi) d t \geq 0 .
$$


Dividing $\lambda$ and letting $\lambda \rightarrow 0$, we obtain

$$
\int_{0}^{T}\left(\hat{F}-F(x, t,|\mathbf{m}|) \mathbf{m}, \varphi d t \geq 0 \quad \text { for all } \varphi \in L^{\infty}\left(0, T ;\left(L^{s}(\Omega)\right)^{d}\right) .\right.
$$

This implies $\hat{F}=F(x, t,|\mathbf{m}|) \mathbf{m}$. (see in the proof of Thm. 1.1 in [21] page 313).

Theorem 5.9. For all $f \in L^{\infty}\left(0, T ; L^{r}(\Omega)\right)$ that is Lipschitz continuous in time $t$. There exists a pair $(\mathbf{m}, u) \in L^{\infty}(0, T ; W(\operatorname{div}, \Omega)) \times L^{\infty}\left(0, T ; L^{r}(\Omega)\right)$, such that

$$
\begin{aligned}
\int_{0}^{T}(F(x,|\mathbf{m}|) \mathbf{m}, \mathbf{v}) d t-\int_{0}^{T}(\nabla \cdot \mathbf{v}, u)=0 & \text { for all } \mathbf{v} \in L^{1}\left(0, T ; L^{r}(\Omega)\right), \\
\int_{0}^{T}\left(\phi \partial_{t} u^{\lambda}, q\right) d t+\int_{0}^{T}(\nabla \cdot \mathbf{m}, q) d t=\int_{0}^{T}(f, q) d t & \text { for all } q \in L^{1}\left(0, T ; L^{r}(\Omega)\right) .
\end{aligned}
$$

Proof. Let $\mathbf{m}$ be the limit of $\pi \mathbf{m}_{h}$ and $u$ be the limit of $\pi u_{h}$. Then Lemma 5.7 and Lemma 5.5part (iii) imply that

$$
\int_{0}^{T}(F(x, t,|\mathbf{m}|) \mathbf{m}, \mathbf{v}) d t=\int_{0}^{T}(\hat{F}, \mathbf{v}) d t=-\int_{0}^{T}(\nabla u, \mathbf{v}) d t=\int_{0}^{T}(u, \nabla \cdot \mathbf{v}) d t
$$

for all $\mathbf{v} \in L^{1}\left(0, T ; L^{r}(\Omega)\right)$. In Lemma 5.6 we have seen that $(\mathbf{m}, u)$ fulfills the second equation. $\square$

\section{REFERENCES}

[1] Y. Amirat, Écoulements en milieu poreux n'obéssant pas à la loi de Darcy, ESAIM: Mathematical Modelling and Numerical Analysis - Modéisation Mathématique et Analyse Numéique, 25 (1991), pp. 273-306.

[2] E. Aulisa, L. Bloshanskaya, L. Hoang, and A. Ibragimov, Analysis of generalized Forchheimer flows of compressible fluids in porous media, J. Math. Phys., 50 (2009), pp. 103102, 44.

[3] H. Brézis, Opérateurs maximaux monotones et semi-groupes de contractions dans les espaces de Hilbert, North-Holland Publishing Co., Amsterdam, 1973. North-Holland Mathematics Studies, No. 5. Notas de Matemática (50).

[4] F. Brezzi and M. Fortin, Mixed and Hybrid Finite Element Methods, Springer Series in Computational Mathematics, Springer New York, 2012.

[5] E. Celik, L. Hoang, A. Ibragimov, and T. Kieu, Fluid flows of mixed regimes in porous media, Journal of Mathematical Physics, 58 (2017), p. 023102.

[6] E. Celik, L. Hoang, And T. Kieu, Doubly nonlinear parabolic equations for a general class of Forchheimer gas flows in porous media, Nonlinearity, 31 (2018), pp. 3617-3650.

[7] E. DiBenedetto, Degenerate parabolic equations, Universitext, Springer-Verlag, New York, 1993.

[8] P. FABRIE, Regularity of the solution of darcy-forchheimer's equation, Nonlinear Anal., 13 (1989), pp. 1025-1049.

[9] L. Hoang ANd A. Ibragimov, Structural stability of generalized Forchheimer equations for compressible fluids in porous media, Nonlinearity, 24 (2011), pp. 1-41.

[10] - Qualitative study of generalized Forchheimer flows with the flux boundary condition, Adv. Diff. Eq., 17 (2012), pp. 511556.

[11] A. Ivanov, P. MKrTychan, AND W. JäGER, Existence and uniqueness of a regular solution of the cauchy-dirichlet problem for a class of doubly nonlinear parabolic equations, Journal of Mathematical Sciences, 84 (1997), pp. 845-855.

[12] A. V. Ivanov, Second-order quasilinear degenerate and nonuniformly elliptic and parabolic equations, Trudy Mat. Inst. Steklov., 160 (1982), p. 285.

[13] A. V. Ivanov, Regularity for doubly nonlinear parabolic equations, Journal of Mathematical Sciences, 83 (1997), pp. 22-37.

[14] T. KIEU, Existence of a solution for generalized Forchheimer flow in porous media, (2018).

[15] P. KNABNer AND G. Summ, Solvability of the mixed formulation for Darcy-Forchheimer flow in porous media, (2017). manuscript. 
[16] O. A. Ladyženskaja, V. A. Solonnikov, and N. N. Ural'ceva, Linear and quasilinear equations of parabolic type, Translated from the Russian by S. Smith. Translations of Mathematical Monographs, Vol. 23, American Mathematical Society, Providence, R.I., 1968.

[17] O. A. Ladyzhenskaya and N. N. Ural'tseva, Linear and quasilinear elliptic equations, Translated from the Russian by Scripta Technica, Inc. Translation editor: Leon Ehrenpreis, Academic Press, New York, 1968.

[18] G. M. Lieberman, Second order parabolic differential equations, World Scientific Publishing Co. Inc., River Edge, NJ, 1996.

[19] J.-L. Lions, Quelques méthodes de résolution des problèmes aux limites non linéaires, Dunod, 1969.

[20] M. Muskat, The flow of homogeneous fluids through porous media, McGraw-Hill Book Company, inc., 1937.

[21] P. Raviart, Sur la résolution de certaines equations paraboliques non linéaires, Journal of Functional Analysis, 5 (1970), pp. $299-328$.

[22] SANDRI, D., Sur lápproximation numérique des écoulements quasi-newtoniens dont la viscosité suit la loi puissance ou la loi de carreau, ESAIM: M2AN, 27 (1993), pp. 131-155.

[23] R. E. ShowaLter, Monotone operators in Banach space and nonlinear partial differential equations, vol. 49 of Mathematical Surveys and Monographs, American Mathematical Society, Providence, RI, 1997.

[24] J. C. WARD, Turbulent flow in porous media., Journal of the Hydraulics Division, Proc. Am. Soc. Civ. Eng., 90(HY5) (1964), pp. 1-12.

[25] E. ZeIDLeR, Nonlinear functional analysis and its applications. II/B, Springer-Verlag, New York, 1990. Nonlinear monotone operators, Translated from the German by the author and Leo F. Boron.

[26] E. Zeidler and L. Boron, Nonlinear Functional Analysis and Its Applications: Part 2 B: Nonlinear Monotone Operators, Nonlinear Functional Analysis and Its Applications, Springer New York, 1989. 